\title{
Overcoming synthesizer phase noise in quantum sensing
}

\author{
by
}

\author{
Guofei Long
}

\author{
A thesis \\ presented to the University of Waterloo \\ in fulfillment of the \\ thesis requirement for the degree of \\ Master of Applied Science \\ in \\ Electrical and Computer Engineering
}

Waterloo, Ontario, Canada, 2019

(C) Guofei Long 2019 
I hereby declare that I am the sole author of this thesis. This is a true copy of the thesis, including any required final revisions, as accepted by my examiners.

I understand that my thesis may be made electronically available to the public. 


\begin{abstract}
Synthesizers are widely used in various quantum information platforms as microwave or radio frequency signal sources. The synthesizer phase noise plays a sensitive role in detecting spin dynamics which is similar to the environment dephasing. When using spins to measure the environmental magnetic field, synthesizer phase noise reduces the accuracy of the measurement because it is difficult to distinguish the effective field caused by the phase noise from the environmental field.

Suppressing the synthesizer phase noise is important in sensing. This work proposes a scheme to suppress the phase noise from synthesizers using two single-spin systems in opposite static magnetic fields. The two spins are exposed to the same environment magnetic field which is to be sensed, and controlled by the same synthesizer.

Two configurations of the scheme are constructed: one uses two antennas for control and detection and the other uses one antenna. Because the two spins experience the phase noise in opposite ways, the phase noise effect can be either canceled or separated from that of the environmental field. While the scheme is based on the Ramsey fringe sequence, it can be extended to the Hahn echo and the stimulated echo sequences as well. The basic idea of the scheme in principle works for different quantum information platforms where the Zeeman interaction is the dominant Hamiltonian.

Nuclear magnetic resonance(NMR) platform is used to experimentally simulate the sensing process using the one-antenna configuration, where the information of the environment field is encoded in the amplitude of the NMR signal, and thus separated from the synthesizer phase noise. The experiment demonstrated successfully the proposed scheme.
\end{abstract}




\section{Acknowledgements}

I would like to thank all my family members who support me all the time. I especially thank my supervisors Prof. Cory and Prof. Miao. They provide me a great chance to study with talented people. I felt unconfident at the beginning, but their kindness and patience made me get there at last. I need to say thank you to my roommates and my friends Duan Bojia, Zhu Yuanye, Zhu Zhipeng, Guo Chuhui, Liu Yu, Wang Hanyang, and Su Hang. Thanks a lot to Carly Turnbull and Lorna Kelly for helping me with the degree completion process. Then I want to say thank you to Chen Jiahui, Feng Guanru and Peter Sprenger who support me on my study and on my mental health. At last, I want to say thank you to my cat Gizmo. He stays with me all the time and makes me keep going. 


\section{Dedication}

This is dedicated to the one I love. 


\section{Table of Contents}

List of Tables $\quad$ viii

List of Figures $\quad$ ix

1 Introduction $\quad 1$

2 Nuclear Magnetic Resonance system $\quad 6$

2.1 Basic principles of NMR system . . . . . . . . . . . . . . . . 7

2.2 Hamiltonian in NMR system . . . . . . . . . . . . . . . . . . . . . 8

2.3 Measurement in NMR system . . . . . . . . . . . . . . . . . 11

2.4 Advantages and disadvantages . . . . . . . . . . . . . . . . . . . 13

$\begin{array}{lll}3 & \text { Scheme for eliminating the synthesizer phase noise } & 17\end{array}$

3.1 Ramsey experiment . . . . . . . . . . . . . . . . . . . . . . . . 17

3.2 Synthesizer noise in Ramsey experiment . . . . . . . . . . . . . . 18

3.3 Methods to suppress synthesizer phase noise . . . . . . . . . . . . . . . 19

3.3 .1 Two-antenna method . . . . . . . . . . . . . . . . . . . 21

3.3 .2 One-antenna method . . . . . . . . . . . . . . . . . . . . . 24

3.4 Hahn echo sequence and stimulated echo sequence . . . . . . . . . . . 25

3.4 .1 Hahn echo sequence. . . . . . . . . . . . . . . 25

3.4 .2 Stimulated echo sequence . . . . . . . . . . . . 26

3.4.3 Extending to Hahn echo and Stimulated echo sequence . . . . . . 27 
4 Experiment $\quad 29$

4.1 Experiment preparation . . . . . . . . . . . . . . . . 29

4.2 Data analysis . . . . . . . . . . . . . . . . 30

4.2.1 Experiments without $B_{\alpha} \ldots \ldots \ldots$. . . . . . . . . 31

4.2 .2 Experiments with $B_{\alpha} \ldots \ldots \ldots$. . . . . . . . . . 34

5 Conclusions and Discussions $\quad 42$

$\begin{array}{ll}\text { References } & 44\end{array}$ 


\section{List of Tables}

1.1 Time until a qubit physical error rate $p$ is reached solely due to phase fluctuations in the synthesizer (reproduced from [46].) . . . . . . . . . 2 


\section{List of Figures}

1.1 Amplitude noise and phase noise. . . . . . . . . . . . . . . 3

2.1 The molecular structure of labeled chloroform. Here, "label" means ${ }^{12} \mathrm{C}$ with nuclear spin -0 replaced by ${ }^{13} \mathrm{C}$ with nuclear spin $-1 / 2$. So ${ }^{1} \mathrm{H}$ and ${ }^{13} \mathrm{C}$ can be used as qubits. . . . . . . . . . . . . . . . .

2.2 Schematic of a standard NMR system. The magnet and the console are main parts of the whole system. The magnet is superconducting magnet and console has the electronics devices to control the spectrometer. The liquid sample is inserted into the central of the magnet, where it is surrounded by coils which we call probe. And radio frequency $(\mathrm{RF})$ pulse can be applied in the probe to control the spins. . . . . . . . . . . . . . 14

2.3 Energy diagram of a single spin-1/2. . . . . . . . . . . . . . 15

2.4 Precession of a spin-1/2 particle in a static magnetic field. . . . . . . . . . 15

2.5 Energy level diagram. dashed lines for two uncoupled spins, and solid lines for two spins coupled by a Hamiltonian $H_{J}=\hbar \sum_{i<j}^{n} 2 \pi J_{i j} I_{z}^{i} I_{z}^{j} \cdot \quad \ldots \ldots . . \quad 16$

3.1 Ramsey sequence contains two $\pi / 2$ pulses the time t are same value. . . . 18 
3.2 The two-pulse Ramsey sequence. It can be used to detect an offset magnetic field $B_{\alpha}$ in the environment. However, the synthesizer phase noise effectively adds a noise term $B_{n}$. In a Ramsey experiment, the initial state is the thermal equilibrium state $\sigma_{z}$ and the final state after the second $\pi / 2$ rotation is $\cos \left(\theta_{\alpha}-\theta_{n}\right) \sigma_{z}+\sin \left(\theta_{\alpha}-\theta_{n}\right) \sigma_{y}$ (the two rotations are assumed to be along $y$-axis). The second $\pi / 2$ rotation is used to restore the spin polarization to $z$-axis for detection. Here $\theta_{\alpha}$ and $\theta_{n}$ are the phases induced by $B_{\alpha}$ and the synthesizer noise, respectively. These two phases acquired by the spin are difficult to separate. . . . . . . . . . . . . . . . .

3.3 Ramsey sequence when spin polarizations in $x y$-plane can be detected. The second $\pi / 2$ rotation as in Figure. 3.2 can be neglected. . . . . . . . . . . .

3.4 The two-antenna configuration (a), and one-antenna configuration (b) of the proposed scheme. Here multi-round coils are used to represent antennas. Two single-spin systems are placed in two opposite static magnetic fields, $B_{0}$ and $-B_{0}$. They are controlled using the same synthesizer and exposed to the same environment offset field $B_{\alpha}$ which is to be sensed. . . . . . . .

3.5 Hahn echo is similar to Ramsey sequence. But the second $\pi / 2$ pulse is replaced by a $\pi$ pulse, so Hahn echo contains one $\pi / 2$ pulse and one refocusing

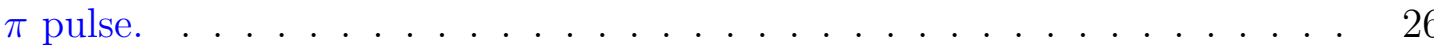

3.6 Stimulated echo contains three $\pi / 2$ pulse, $t$ and $T$ are different value. . . .

3.7 Hahn echo sequence. The initial state of a Hahn echo experiment is usually the thermal equilibrium state. Here, we assume the environment offset field $B_{\alpha}$ is not present after the $\pi$ refocusing pulse. . . . . . . . . . . . . .

3.8 Stimulated echo sequence. The initial state of a stimulated echo experiment is usually the thermal equilibrium state. Here, we assume the environment offset field $B_{\alpha}$ is not present after the third $\pi / 2$ pulse. . . . . . . . . . . .

4.1 Chloroform molecule structure. The ${ }^{13} \mathrm{C}$ and ${ }^{1} \mathrm{H}$ are spin-half nuclei. Their chemical shifts and $J$-coupling constant are listed as the diagonal and offdiagonal elements in the table. The topmost panel shows a thermal spectrum of ${ }^{1} \mathrm{H}$, where the two peaks associate with the two Zeeman eigenstates, spinup and spin-down, of ${ }^{13} \mathrm{C}$ in a magnetic field. . . . . . . . . . . . . 30

4.2 The noise power density spectrum used for noise generation. . . . . . . . . 31 
4.3 FID in the $\mathrm{H}$ rotating frame. Blue line represents the real part of FID and the red line is the imaginary part of FID. As shown in the two Figures the real parts are similar but the imaginary part seems cancel out for two-spin system as can be explained by Eq. (3.8). . . . . . . . . . . . . . . . . . .

4.4 Experimental frequency spectra. No offset field is present. (a) is the spectrum of the experiment where two spins in two magnetic fields with $\pm \omega_{0}=$ $\pm 107.6 \mathrm{~Hz}$ are simulated. (b) is the spectrum of the experiment where one spin in one magnetic field with $\omega_{0}=107.6 \mathrm{~Hz}$ is simulated for comparison with $(\mathrm{a}) \ldots \ldots \ldots \ldots \ldots \ldots$

4.5 Experimental FID after demodulation with perfect synthesizer signal. No offset field is present. (a) and (b) are the demodulated FID signals of Figure.4.3(a) and Figure.4.3(b), respectively. The numerically generated perfect synthesizer signal $\cos \left(\omega_{0} t\right)$ is used for demodulation. In (a) and (b), the real, imaginary and absolute signals of the demodulated FIDs are in blue, red and green, respectively. The real and absolute signals overlap a lot due to the small imaginary signals. Both the FIDs decay due to the $T_{2}^{*}$. . . .

4.6 Experimental FID after demodulation with noisy synthesizer signal. No offset field is present. (a) and (b) are the demodulated FID signals of Figure.4.3(a) and Figure.4.3(b), respectively. The numerically generated perfect synthesizer signal $\cos \left(\omega_{0} t\right)$, with injected phase noise (Figure. 4.2), is used for demodulation. In (a) and (b), the real, imaginary and absolute signals of the demodulated FIDs are in blue, red and green, respectively. The real and imaginary signals of both the two spectra are very noisy because of the phase noise of the synthesizer signal. Both the FIDs decay due to the $T_{2}^{*} \cdot 34$

4.7 The Fourier transform spectra of the FIDs after demodulation with phase noise as in Figure. 4.6. The major peaks are at zero frequency because that there is no offset field. . . . . . . . . . . . . . . . . . . . .

4.8 Average FIDs over 500 processings after demodulation with phase noise. (There is signal loss due to averaging, but the noise is averaged to almost zero.) . . . . . . . . . . . . . . . . . . . 35

4.9 The Fourier transform spectra of the average FIDs as shown in Figure. 4.8. 36 
4.10 The FIDs in the $\mathrm{H}$ rotating frame of the two-spin and one-spin experiments when there is an offset field. Absolute, real and imaginary values are in green, blue and red, respectively. In the two-spin experiment, the amplitude of the signal oscillates, while in the one-spin experiment only real and imaginary parts of the signal oscillate. Those oscillations are around the frequency $\omega_{0} \ldots \ldots \ldots \ldots \ldots \ldots$

4.11 The Fourier transform spectra of the FIDs in Figure. 4.11. (a) is the spectrum of the experiment where two spins in two magnetic fields with $\pm \omega_{0}= \pm 107.6 \mathrm{~Hz}$ are simulated but the frequency shift to positive side compare to Figure. 4.4 (a). (b) is the spectrum of the experiment where one spin in one magnetic field with $\omega_{0}=107.6 \mathrm{~Hz}$ but also shift to the positive side compare to Figure. 4.4 (b)is simulated for comparison with (a).

4.12 Experimental FID after demodulation in presence of an offset field $B_{\alpha}$ with $\omega_{\alpha}=10 \mathrm{~Hz}$. (a) and (c) are the FIDs from the experiment where two spins in two magnetic fields with $\pm \omega_{0}= \pm 107.6 \mathrm{~Hz}$ are simulated. (b) and (d) are the FIDs from the experiment where one spin in one magnetic field with $\omega_{0}=107.6 \mathrm{~Hz}$ is simulated for comparison. (a) and (b) are demodulated using the perfect synthesizer signal $\cos \left(\omega_{0} t\right)$. (c) and (d) are demodulated using the synthesizer signal with injected phase noise, $\cos \left(\omega_{0} t+\phi(t)\right)$. The real, imaginary and absolute signals of the demodulated FIDs are in blue, red and green, respectively. In (a), the absolute signal oscillates as $\left|\cos \left(\theta_{\alpha}\right)\right|$. In (c), although there is phase noise in demodulation, this oscillation remains. In (b), the real and imaginary signals oscillate as $\cos \left(\theta_{\alpha}\right)$ and $\sin \left(\theta_{\alpha}\right)$. After introduction of the phase noise, the oscillations are concealed by the noise

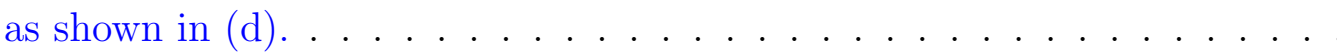

4.13 The Fourier transform spectra of the FIDs in Figure.4.12(c,d). The major peaks at $\pm 10 \mathrm{~Hz}$ come from the offset field, and the noisy small peaks come from the synthesizer phase noise. . . . . . . . . . . . . . .

4.14 The average of 500 copies of the FIDs after demodulation with phase noise. (There is signal loss due to averaging, but the noise is averaged to almost zero.) . . . . . . . . . . . . . . . . . .

4.15 The Fourier transform spectra of the average FIDs as shown in Figure. 4.14. The noise disappears and only the major peaks remain. The major peaks have a frequency of $\pm \omega_{0} \ldots \ldots$. . . . . . . . . . . . . . 


\section{Chapter 1}

\section{Introduction}

In 1982, Feynman pointed out that the use of classical computers to simulate quantum systems will have some impassable problems, which could be avoided by constructing computers based on quantum mechanics [1]. In the same year, Benioff proposed that a Turing machine can be constructed by using quantum mechanics. Since the evolution of the Hamiltonian is reversible, there is no heat dissipation in quantum Turing machine [2]. In 1985, Deutsch defined a universal computer based on the principles of quantum mechanics [3].

Many quantum algorithms were published over the next two decades. The most famous two were proposed by Peter Shor [4] and Lov Grover [5, 6]. Shor's algorithm suggested that quantum computers are more powerful than classical computers for integer factorization. Grover's searching algorithm attracted many attention [7, 8, 9, 10, 11, 12], because it has many applications, although the algorithm does not reach the speedups of Shor's algorithm. Additional research on quantum algorithms motivated by Shor's algorithm and Grover's algorithm have been reported [13, 14, 15, 16, 17, 18, 19, 20, 21, 22, 23, 24, 25, 26]. Research on quantum simulations also has progressed with many achievements such as simulations of fermion many-body system [27], quantum harmonic oscillator [28], quantum phase transition [29, 30, 31, 32], and Dirac equations [33]. While studying the standard quantum computer proposed by Deutsch [34], researchers are also exploring other quantum computing models, such as topological quantum computing [35, 36, 37, 38, 39], adiabatic quantum computing [40, 41], one-way quantum computing [42], etc.

There is another very important field in research of quantum computing, which is quantum error correction and fault tolerance [43, 44, 45]. Because the quantum state is very fragile itself, the interaction between the quantum bit and the environment will cause de- 


\begin{tabular}{|cccccc|}
\hline \multicolumn{6}{|c|}{ Time to Reach Synthesizer-Induced Error Rate $p$} \\
\hline$p$ & $10^{-3}$ & $10^{-4}$ & $10^{-5}$ & $10^{-6}$ & $10^{-7}$ \\
Lab-Grade Synthesizer & $4.0 \mu \mathrm{s}$ & $900 \mathrm{~ns}$ & $200 \mathrm{~ns}$ & $30 \mathrm{~ns}$ & $<10 \mathrm{~ns}$ \\
Precision Synthesizer & $>100 \mathrm{~ms}$ & $>100 \mathrm{~ms}$ & $>100 \mathrm{~ms}$ & $80 \mathrm{~ms}$ & $600 \mu \mathrm{s}$ \\
\hline
\end{tabular}

Table 1.1: Time until a qubit physical error rate $p$ is reached solely due to phase fluctuations in the synthesizer (reproduced from [46].)

coherence, which will transfer quantum states into classical states. On the other hand, the errors will occur in quantum operations which will lead to the failure of quantum computing. Quantum threshold theory states that as long as the physical error rate is below a certain threshold, with quantum error correction the logical error rate in quantum computing can be suppressed to arbitrary low levels. Quantum error correction is important to realize successful quantum computing if one has faulty memories, faulty operations and faulty measurements which are inevitable. The research on quantum error correction codes and fault tolerance make the physical implementation of quantum computing accessible $[47,48,49,50,51,52]$.

In addition to the general-purpose quantum computers, special-purpose quantum devices have also attracted great interest, one of which is quantum sensors. Different from classical sensing, quantum sensing uses quantum properties or quantum phenomena to implement measurement of a quantity properties or quantity [53]. At first superconducting quantum interference devices [54], atomic vapors [55] and atomic clocks [56] are chosen to achieve quantum sensors. Recently many new types of quantum sensors come out from the following platforms: spin qubits [57], trapped ions [58] and superconducting flux qubits.

Although great improvements have been made on different platforms [59, 60, 61, 62], one error source has been long ignored by the quantum information society until recently [46], which is the phase noise of master clocks, i.e. the synthesizer phase noise, that contributes to dephasing. Time constants for reaching different error rate of Lab-Grade synthesizers and precision synthesizer are shown in Table 1.1. Different quantum platforms, e.g., quantum dot systems, ion trap systems, nuclear and electron magnetic resonance systems, utilize synthesizers for microwave and radio frequency signal generation. Synthesizer phase noise is a common problem in all those systems.

Although radio-frequency and microwave technologies are well-developed, phase noise is still a big problem which can affect the accuracy of the experiment.

Phase noise is the result of a random fluctuation of the frequency output of the syn- 
thesizer and one of the factors to be concerned within modern quantum devices. We use NMR to demonstrate the effect of phase noise on a quantum gate and suggest ways to eliminate it. Amplitude fluctuation and phase fluctuation are two different types of noise

\section{Time domain}
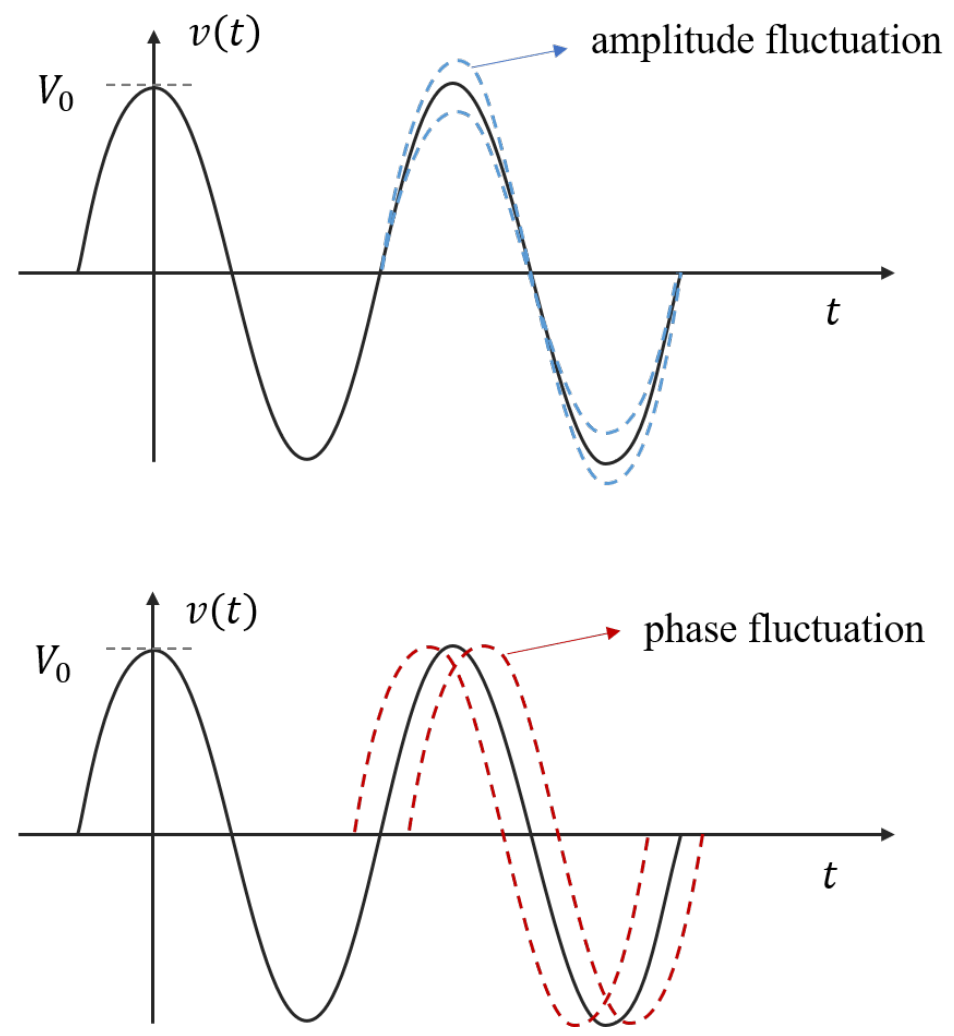

Figure 1.1: Amplitude noise and phase noise.

on oscillators. We denote amplitude fluctuation as $V_{0} \alpha(t)$, and phase fluctuation as $\phi(t)$. An oscillator signal with amplitude and phase fluctuations can be written as:

$$
v(t)=V_{0}[1+\alpha(t)] \cos \left[\omega_{0} t+\phi(t)\right], \quad|\alpha(t)| \ll 1, \quad|\phi(t)| \ll 1
$$

In this thesis, only phase fluctuation is discussed.

Dephasing is one important error source of decoherence. It is always attributed to fluctuations of the environmental fields. However, as discussed in [46], the phase noise of the reference signal from the synthesizer plays a role which is indistinguishable from the 
environment dephasing. It is because the measurement and control of the coherence of a spin system have to be compared to the reference signal. As the spin coherence and control fidelity improve, the error from the synthesizer noise will become more prominent. Besides, demand for miniaturization of quantum information processors requires signal sources of small sizes, which may have larger phase noise due to the lack of stabilizing/filtering components. Therefore, for achieving good accuracy in quantum information processing, overcoming the synthesizer phase noise is one important challenge.

Different approaches can be considered to suppress the synthesizer noise. Efforts on building synthesizers with higher accuracy have been going all the time.

Besides, a feedback/feedforward hybrid control method which utilizes multiple measurements is used to reduce the synthesizer noise [63]. Sastrawan et al introduced introduce a measurement protocol which is an optimized hybrid predictive feedforward measurement. This feedforward measurement can improve the accuracy of the corrections in feedback loop by using past measurements and transfer-function-based calculation of the covariance of measurement.

Furthermore, there have been existing dynamical decoupling pulse sequences for eliminating noise from environmental fluctuations. Gordon and Kurizki proposed a theory of dynamical control for the reduction of decoherence[64].

A general constructive procedure is presented by Khodjasteh and Viola, which is for designing robust unitary gates in an open quantum system[65]. Khodjasteh and his colleague showed that in open quantum systems, open-loop dynamical control techniques is a promising way to compensate the decoherence up to a higher level [66].

Souza and his colleagues investigated techniques for increasing the robustness of dynamical decoupling sequences against different experimental errors[67].

These works may help for suppressing the synthesizer phase noise. However, the farfrom-carrier frequency components of the synthesizer noise can violate the decoupling limit and make dynamical decoupling fail [46].

Other optimal control techniques, e.g. gradient ascent pulse engineering(GRAPE) [68], and quantum error correction [69] can as well be potential choices to overcome the synthesizer phase noise, but they are either requiring complicated optimization or complicated quantum gate operations that are difficult to realize with current technologies.

In this work, we focus on the important modality - quantum sensing. The goal of this work is to detect an environment magnetic field accurately by spins in the presence of the synthesizer phase noise. Here we assume that the spin has long coherence times: the environment is very clean except for the magnetic field that we want to detect. In this 
context, it is relatively difficult to design optimal control pulses, because a sequence robust to the synthesizer noise is likely to be insensitive to the environmental magnetic field due to their similar coupling forms with the spin in the rotating frame. In the following, we propose a scheme to completely eliminate the effect of the synthesizer phase noise in sensing by utilizing two single-spin systems in opposite static magnetic fields. The two spins which are controlled by the same synthesizer experience the synthesizer noise in opposite ways and thus the phase noise can be cancelled. This approach works generally for all qubit systems which utilize a RF or microwave signal source for control and have the Zeeman interaction as the dominant Hamiltonian. More specifically, two configurations, one with two antennas for control and detection and the other with only one antenna, are proposed in this work. The phase noise effect is removed from the measurement of the environment in slightly different ways in the two configurations. We begin by a theoretical analysis of the scheme based on the Ramsey sequence. Hahn echo and stimulated echo sequences are also discussed. Then an NMR experimental demonstration of the one-antenna configuration is presented.

My thesis will focus on suppressing the phase noise in the synthesizer. In chapter 2, I will introduce the experiment platform NMR system and Ramsey sequence. In chapter 3, I will introduce my proposal using two spins in two opposite magnetic fields. In chapter 4, I will introduce our simulation experiments using an NMR system. In chapter 5, I provide directions for future research. 


\section{Chapter 2}

\section{Nuclear Magnetic Resonance system}

In this chapter, the experiment platform nuclear magnetic resonance (NMR) system is introduced. The basic principles of NMR system, including its internal and control Hamiltonians, are reviewed. This chapter provides basic knowledge for the next two chapters.

NMR uses the interaction between electromagnetic waves and the nuclei of atoms. NMR was first discovered in 1946 by Purcell and Bloch [70, 71]. They found that the magnetic nucleus which is exposed to the strong magnetic field can interact with radio frequency (about $10 \mathrm{M} \mathrm{Hz}$ to $1000 \mathrm{M} \mathrm{Hz}$ ) electromagnetic waves. A magnetic nucleus can absorb the energy from electromagnetic waves and produce resonance absorption signals due to transitions between different energy levels.

Nuclear magnetic resonance as a research method, in physics, chemistry, chemical engineering, biology, medicine, and other fields has very important applications.

Cory and his colleagues[73], Gershenfeld and Chuang[74], first gave the proposals for doing quantum information on liquid-state NMR systems. The quantum information processing by NMR system is similar to the analysis of unknown molecular structures by NMR, and many techniques of the latter can be applied to NMR quantum information processing. But there is a crucial difference. The NMR quantum information processing uses molecules that have been specially designed or clearly structured so that the information can be loaded onto the nuclear spins of these molecules and the nuclear spins can be precisely controlled using NMR techniques. 


\subsection{Basic principles of NMR system}

Many atomic nuclei have spin momentum. The three isotopes of hydrogen, H, D, and T, for example, all have spins because they have an odd number of protons, and the spin quantum numbers are 1/2, 1 and 1 , respectively. The isotope of carbon ${ }_{6}^{13} \mathrm{C}\left({ }^{13} \mathrm{C}\right)$ has an even number of protons and an odd number of neutrons, so it also has a spin with a spin quantum number of $1 / 2$.

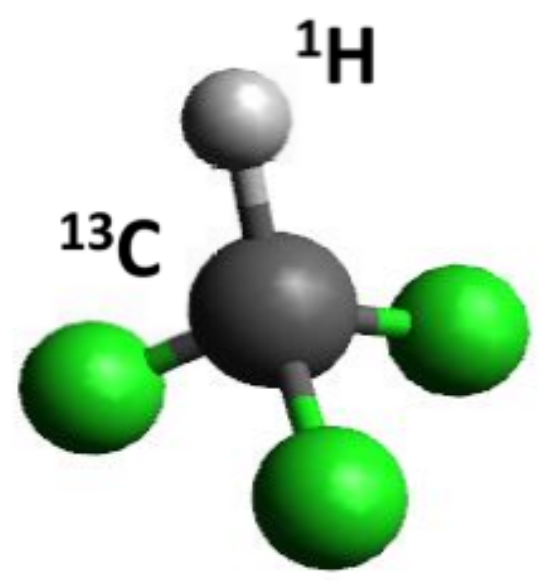

Figure 2.1: The molecular structure of labeled chloroform. Here, "label" means ${ }^{12} \mathrm{C}$ with nuclear spin -0 replaced by ${ }^{13} \mathrm{C}$ with nuclear spin $-1 / 2$. So ${ }^{1} \mathrm{H}$ and ${ }^{13} \mathrm{C}$ can be used as qubits.

If the nuclei are exposed to an external magnetic field, their spin magnetic moments interact with the field, allowing them to be detected by this interaction. Although measuring the magnetic moment of a single nucleus is difficult at present, when enough atoms are present, the effect of the spin magnetic moment on the external magnetic field of all nuclei can be observed by summation. Such a group of atomic nuclei is called an ensemble.

We take liquid nuclear magnetic resonance (NMR) as an example. Suppose that we focus on the nucleus ${ }^{13} \mathrm{C}$. The molecular structure of chloroform is shown in Figure. 2.1. Each chloroform molecules contains a ${ }^{1} \mathrm{H}$ and a ${ }^{13} \mathrm{C}$ atoms, and their nuclear spin quantum number is $1 / 2$. Some amount of chloroform molecules is dissolved in the solvent, and used as a liquid sample. The whole sample is placed in the external magnetic field in 
an equilibrium state. The net result of the ${ }^{1} \mathrm{H}$ nuclear magnetic moment (or all ${ }^{13} \mathrm{C}$ nuclear magnetic moment) tends to be arranged along the external magnetic field direction.

A radio frequency magnetic field rotates the spin. This precession frequency, $\nu$, is the Larmor frequency, and is about the $\hat{z}$ axis. The gyromagnetic ratio $\gamma$ of the nuclear spin, where $\nu=\gamma B / 2 \pi$, governs the rule of spin precession.

\subsection{Hamiltonian in NMR system}

In section 2.1, we briefly introduced the basic principles and physical images of NMR systems. In this section, we will introduce the Hamiltonians under which the NMR systems evolve. The Hamiltonian $H$ of an NMR quantum system is usually divided into three parts: the internal Hamiltonian $H_{i n}$, the control Hamiltonian $H_{c o n}$, and the Hamiltonian $H_{e n v}$, which is the interaction of the nuclear spins with the environment:

$$
H=H_{i n}+H_{c o n}+H_{e n v} .
$$

\section{Internal Hamitltonian}

Firstly, the internal Hamiltonian $H_{\text {in }}$ is introduced. So let us just think the system has nuclei with spin $1 / 2, H_{\text {in }}$ only contains the Zeeman interaction $H_{Z}$ between the nuclear spin and the static magnetic field:

$$
H_{Z}=-\vec{\mu} \cdot \vec{B}
$$

where $\vec{\mu}$ is the nuclear magnetic moment, and $\vec{B}$ is the external static magnetic field. In general, the direction of the static magnetic field will be selected as the z-axis, that is, $\vec{B}=B_{0} \hat{z}$, so the above equation can be further written as:

$$
H_{Z}=-\hbar \gamma B_{0} I_{z}=-\hbar \omega_{0} I_{z}=\frac{1}{2}\left(\begin{array}{cc}
-\hbar \omega_{0} & 0 \\
0 & \hbar \omega_{0}
\end{array}\right)
$$

where $\gamma$ is the gyromagnetic ratio (the ratio of the nuclear magnetic moment to the spin momentum moment), $\omega_{0} /(2 \pi)$ is the Larmor frequency, and $I_{z}$ is the nuclear spin operator in the direction of $\hat{z}$. The nuclear spin operators for the $x$ and $y$ directions are $I_{x}$ and $I_{y}$, respectively. The matrix forms of $I_{x}, I_{y}$ and $I_{z}$ are as follows:

$$
I_{x}=\frac{1}{2} \sigma_{x}=\frac{1}{2}\left(\begin{array}{ll}
0 & 1 \\
1 & 0
\end{array}\right), I_{y}=\frac{1}{2} \sigma_{y}=\frac{1}{2}\left(\begin{array}{cc}
0 & -i \\
i & 0
\end{array}\right), I_{z}=\frac{1}{2} \sigma_{z}=\frac{1}{2}\left(\begin{array}{cc}
1 & 0 \\
0 & -1
\end{array}\right) .
$$


For nuclear spins in molecules, there are two types of interactions, the direct dipoledipole interaction and $J$-coupling interaction.

The first important interaction is the direct coupling. To feel how the magnetic dipoledipole interaction works, one can try to find two magnet bars and play with them. This interaction does not need any media, it only depends on the distance between the nuclei $r_{i j}$, which is the distance between nuclei $i$ and $j$, as described by the following Hamiltonian:

$$
H_{D}=\sum_{i<j} \frac{\mu_{0} \gamma_{i} \gamma_{j} \hbar}{16 \pi\left|\vec{r}_{i j}\right|^{3}}\left[\vec{\sigma}^{i} \cdot \vec{\sigma}^{j}-\frac{3}{\left|\vec{r}_{i j}\right|^{2}}\left(\vec{\sigma}^{i} \cdot \vec{r}_{i j}\right)\left(\vec{\sigma}^{j} \cdot \vec{r}_{i j}\right)\right],
$$

where $\mu_{0}$ is magnetic permeability of free space and $\vec{\sigma}^{i}$ is Pauli matrix of spin $i$.

When the external field is strong, we can take the secular approximation:

$$
H_{D}=\sum_{i<j} \frac{\mu_{0} \gamma_{i} \gamma_{j} \hbar}{32 \pi\left|\vec{r}_{i j}\right|^{3}}\left(1-3 \cos ^{2} \theta_{i j}\right)\left[3 \sigma_{z}^{i} \sigma_{z}^{j}-\vec{\sigma}^{i} \cdot \vec{\sigma}^{j}\right],
$$

where the angle between $B_{0}$ and $\overrightarrow{r_{i j}}$ is $\theta_{i j}$. If the difference of gyromagnetic ratio of two spins is much larger than coupling strength, $H_{D}$ can be rewritten as:

$$
H_{D}=\sum_{i<j} \frac{\mu_{0} \gamma_{i} \gamma_{j} \hbar}{16 \pi\left|\vec{r}_{i j}\right|^{3}}\left(1-3 \cos ^{2} \theta_{i j}\right) \sigma_{z}^{i} \sigma_{z}^{j},
$$

which has the similar form as the $J$-coupling. Rapid tumbling will average away both intramolecular dipolar couplings (between spins in the same molecule) and intermolecular dipolar couplings (between spins in different molecules) in liquid.

The second important interaction is indirect coupling. $J$-coupling describe the interaction between nuclear spins in the same molecule.

$J$-coupling is mediated via valence electrons shared between the atoms, namely the Fermi contact interaction. The numbers of chemical bonds which separate the nuclei affect through-bond coupling strength $J$. With the number of chemical bonds separating the nuclei increases, $J$ decreases. The Hamiltonian is

$$
H_{J}=\hbar \sum_{i<j} \frac{\pi}{2} J_{i j} \vec{\sigma}^{i} \cdot \vec{\sigma}^{j}=\hbar \sum_{i<j} \frac{\pi}{2} J_{i j}\left(\sigma_{x}^{i} \sigma_{x}^{j}+\sigma_{y}^{i} \sigma_{y}^{j}+\sigma_{z}^{i} \sigma_{z}^{j}\right),
$$


where $J_{i j}$ is the coupling strength between spins $i$ and $j$. Similar to the case of Eq.(2.7), Eq. (2.8) can be rewritten to

$$
H_{J}=\hbar \sum_{i<j}^{n} \frac{\pi}{2} J_{i j} \sigma_{z}^{i} \sigma_{z}^{j}
$$

when $\left|\omega_{i}-\omega_{j}\right| \gg 2 \pi\left|J_{i j}\right|$, which is satisfied for small homonuclear molecules and heteronuclear spins. A spin not only feels an external magnetic field $\overrightarrow{B_{0}}$, but also feels another static magnetic field along $\pm \hat{Z}$ axis which is generated by its surrounding spins. Figure. 2.5 shows the energy levels which is shifted by additional field.

In general, for a system of $n$ coupled nuclear spins the simplest form of the Hamiltonian is

$$
H_{i n}=-\sum_{i} \frac{1}{2} \hbar \omega_{0}^{i} \sigma_{z}^{i}+\hbar \sum_{i<j} \frac{\pi}{2} J_{i j} \sigma_{z}^{i} \sigma_{z}^{j}
$$

In almost all NMR quantum computing experiments performed to date, the system is well described by a Hamiltonian of this form.

\section{Control Hamiltonian}

Next, we will discuss the control Hamiltonian $H_{c o n}$. In the NMR system, the applied control pulses are all transverse, that is, the magnetic field direction of the pulse is perpendicular to the direction of the static magnetic field (direction $\hat{z}$ ).

$$
H_{\text {con }}=-\frac{\hbar}{2} \sum_{k=1}^{n} \gamma^{k} B_{1}\left(\cos \left(\omega_{r f} t+\phi\right) \sigma_{x}^{k}-\sin \left(\omega_{r f} t+\phi\right) \sigma_{y}^{k}\right) \text {. }
$$

RF field. An electromagnetic field $\vec{B}_{1}(t)$ rotating in $x-y$ plane at $\omega_{r f}$ (at or close to $\left.\omega_{0}\right)$ can be used to control the state of a spin $-1 / 2$ particle in a static magnetic field $\vec{B}_{0}$ along $\hat{z}$.

The single-spin Hamiltonian of radio-frequency (RF) field is:

$$
H_{r f}=-\frac{1}{2} \hbar \gamma B_{1}\left[\cos \left(\omega_{r f} t+\phi\right) \sigma_{x}+\sin \left(\omega_{r f} t+\phi\right) \sigma_{y}\right]
$$

where $\phi$ is the phase of the RF field, and $B_{1}$ is the amplitude of RF field.

The typical values of $\omega_{1}=\gamma B_{1}$ are up to $\approx 50 \mathrm{kHz}$ and a few hundred $k \mathrm{~Hz}$ for liquid and solid NMR experiments, respectively. For a molecule with $n$ spins, we have 


$$
H_{r f}=-\frac{1}{2} \sum_{i}^{n} \hbar \gamma_{i} B_{1}\left[\cos \left(\omega_{r f} t+\phi\right) \sigma_{x}^{i}+\sin \left(\omega_{r f} t+\phi\right) \sigma_{y}^{i}\right]
$$

In real experiments, a magnetic field which is perpendicular to the static magnetic field is applied. It is oscillating along a fixed axis and this field can be divided into two rotating fields. The first one is rotating at $\omega_{r f}$ in the same direction of spin. The second one is rotating in another direction which is the opposite direction of spin. So the first one can be set on resonance or near resonance with the spin. And the second one is off-resonance about $2 \omega_{0}$.

Rotating frame. For the motion of a single nuclear spin in a static magnetic field and a rotating magnetic field, it is easy to use rotating frame rather than lab frame. A coordinate system rotating along $\hat{z}$ with frequency $\omega_{r f}$ is:

$$
|\psi\rangle^{r o t}=\exp \left(-\frac{i \omega_{r f} t \sigma_{z}}{2}\right)|\psi\rangle
$$

Substitution of $|\psi\rangle$ in $i \hbar \frac{d|\psi\rangle}{d t}=H|\psi\rangle$, where

$$
H=-\frac{1}{2} \hbar \omega_{0} \sigma_{z}-\frac{1}{2} \hbar \omega_{1}\left[\cos \left(\omega_{r f} t+\phi\right) \sigma_{x}+\sin \left(\omega_{r f} t+\phi\right) \sigma_{y}\right] .
$$

gives $i \hbar \frac{d|\psi\rangle^{r o t}}{d t}=H^{r o t}|\psi\rangle^{r o t}$, where

$$
H^{r o t}=-\frac{1}{2} \hbar\left(\omega_{0}-\omega_{r f}\right) \sigma_{z}-\frac{1}{2} \hbar \omega_{1}\left[\cos \phi \sigma_{x}+\sin \phi \sigma_{y}\right] .
$$

This is the interaction of the $n$ nuclear spins with the RF field of intensity $B_{1}, \omega_{r f}$ is the rotation frequency of the RF field in the $x-y$ plane, where $\phi$ is the phase.

The Hamiltonian of an isotropic liquid system with $n$ spin $1 / 2$ nucleus is:

$$
H_{i n}=-\frac{1}{2} \hbar \sum_{k=1}^{n} \omega^{k} \sigma_{z}^{k}+\hbar \sum_{l<k}^{n} \frac{\pi}{2} J_{l k} \sigma_{z}^{l} \sigma_{z}^{k}
$$

\subsection{Measurement in NMR system}

Projection measurements are not possible in an NMR system. In a liquid NMR system, instead of one molecule, a quantum register is defined by the ensemble of many identical 
molecules, and the measured result is the average of the ensemble. More specifically, the measurable physical quantity in the NMR system is the transverse magnetization vector of the sample, that is the magnetization vector in the $\hat{x}-\hat{y}$ plane. Because of the precession of the transverse magnetization vector around the axis $\hat{z}$, the axis induces a current in the coil in the $\hat{x}-\hat{y}$ plane. Since the sample system will eventually return to the thermal equilibrium state, the transverse magnetization vector will eventually reduce to zero, and the induced current in the coil will finally become zero, so this signal is called free induction decay signal (FID). In this process, the interaction between the coil and any nuclear spin is very weak, so the effect of the coil on the nuclear spin quantum state can be ignored. Assuming that the density matrix of the state to be detected is $\rho$, the free-induced decay signal can be written as [75]

$$
S(t) \propto \operatorname{Tr}\left(e^{-i H t / \hbar} \rho e^{i H t / \hbar} \sum_{k=1}^{n}\left(\sigma_{x}^{k}-i \sigma_{y}^{k}\right)\right) .
$$

If we do not consider the attenuation of the signal and a proportional factor related to the number of ensemble molecules, nuclear magnetic moment and other factors, the above equation can be written as:

$$
S(t)=\operatorname{Tr}\left(e^{-i H t / \hbar} \rho e^{i H t / \hbar} \sum_{k=1}^{n}\left(\sigma_{x}^{k}-i \sigma_{y}^{k}\right)\right) .
$$

Take the Fourier transform of $S(t)$, we get the NMR spectrum and information about the quantum state $\rho$.

Now let us look at the form of $S(t)$ and the information that can be derived from systems of one qubit and two qubits, respectively. Typically, the definition of a qubit in an NMR system and the quantum operations are carried out in a logical coordinate system (multi-rotation coordinate system). But we are going to use laboratory coordinates, which means that $\rho$ and the Hamiltonian $H$ are both forms in laboratory coordinates. Since the phase difference between the laboratory coordinate system and the multi-rotation coordinate system is known, the quantum state in the logical coordinate system can be obtained by the coordinate system transformation after obtaining $\rho$. For one qubit system, $H=-\hbar \omega_{0} I_{z}=-\hbar \omega_{0} \sigma_{z} / 2$, and its FID signal is:

$$
\begin{aligned}
S(t) & =\operatorname{Tr}\left(e^{i \omega_{0} \sigma_{z} t / 2} \rho e^{-i \omega_{0} \sigma_{z} t / 2}\left(\sigma_{x}-i \sigma_{y}\right)\right) \\
& =\operatorname{Tr}\left(\rho e^{-i \omega_{0} \sigma_{z} t / 2}\left(\sigma_{x}-i \sigma_{y}\right) e^{i \omega_{0} \sigma_{z} t / 2}\right) \\
& =\operatorname{Tr}\left(\rho e^{i \omega_{0} t}\left(\sigma_{x}-i \sigma_{y}\right)\right)
\end{aligned}
$$


Apply Fourier transform to the above signal, a peak will be obtained at the frequency $\omega_{0} / 2 \pi$, and the amplitude of the peak will be determined by $\operatorname{Tr}\left(\rho\left(\sigma_{x}-i \sigma_{y}\right)\right)$, that is, the expected value $\left\langle\sigma_{x}\right\rangle=\operatorname{Tr}\left(\rho \sigma_{x}\right)$ and $\left\langle\sigma_{y}\right\rangle=\operatorname{Tr}\left(\rho \sigma_{y}\right)$, can be obtained from the real part and the imaginary part of the peak,respectively. You can also measure it by applying a $\pi / 2$ rotation pulse in $\hat{x}$ direction or $\hat{y}$ direction to $\rho$, and then you can measure it, and you can measure the expected value $\left\langle\sigma_{z}\right\rangle=\operatorname{Tr}\left(\rho \sigma_{z}\right)$. And we know that $\rho$ can be expressed in the following form:

$$
\rho=\frac{1}{2}\left(I+\alpha_{x} \sigma_{x}+\alpha_{y} \sigma_{y}+\alpha_{z} \sigma_{z}\right)
$$

where $\alpha_{x}=\left\langle\sigma_{x}\right\rangle, \alpha_{y}=\left\langle\sigma_{y}\right\rangle, \alpha_{z}=\left\langle\sigma_{z}\right\rangle$.

Similar to the single qubit case, the two-qubit density matrix can be expanded into Pauli matrix and the expansion coefficient can be obtained from the spectrum of the FID signal after Fourier transform. In this way, the coefficients of each Pauli matrix expansion term can be extracted from the spectra obtained from each measurement by making multiple measurements of the system and applying a certain reading pulse before each measurement, and then the two-qubit density matrix can be reconstructed. One aspect that is more complicated than the single-qubit case is that a single-bit density matrix can be reconstructed by only two experiments (directly observing $\rho$ and observing $\rho$ after adding a read pulse), and the reconstruction of the two-qubit density matrix requires more experiments. The same principle applies to density matrix reconstruction of more qubits, which will not be repeated here.

\subsection{Advantages and disadvantages}

NMR quantum computing has certain limitations. For example, it is ensemble quantum computing, using pseudo-pure state rather than a true pure state. Moreover, due to the limited frequency resolution, NMR does not have good scalability. However, the NMR system is still indispensable to experimental quantum computing in the study.

NMR is a demonstration and experimental platform for many quantum algorithms. Researchers have verified many basic theories of quantum information and quantum computing. In addition, many technologies developed in NMR quantum computing have, such as spin-echo technology and dynamical decoupling, been adopted in many other experimental systems. In the following chapters, we will introduce specific experiments of quantum simulation using NMR quantum computing system. 


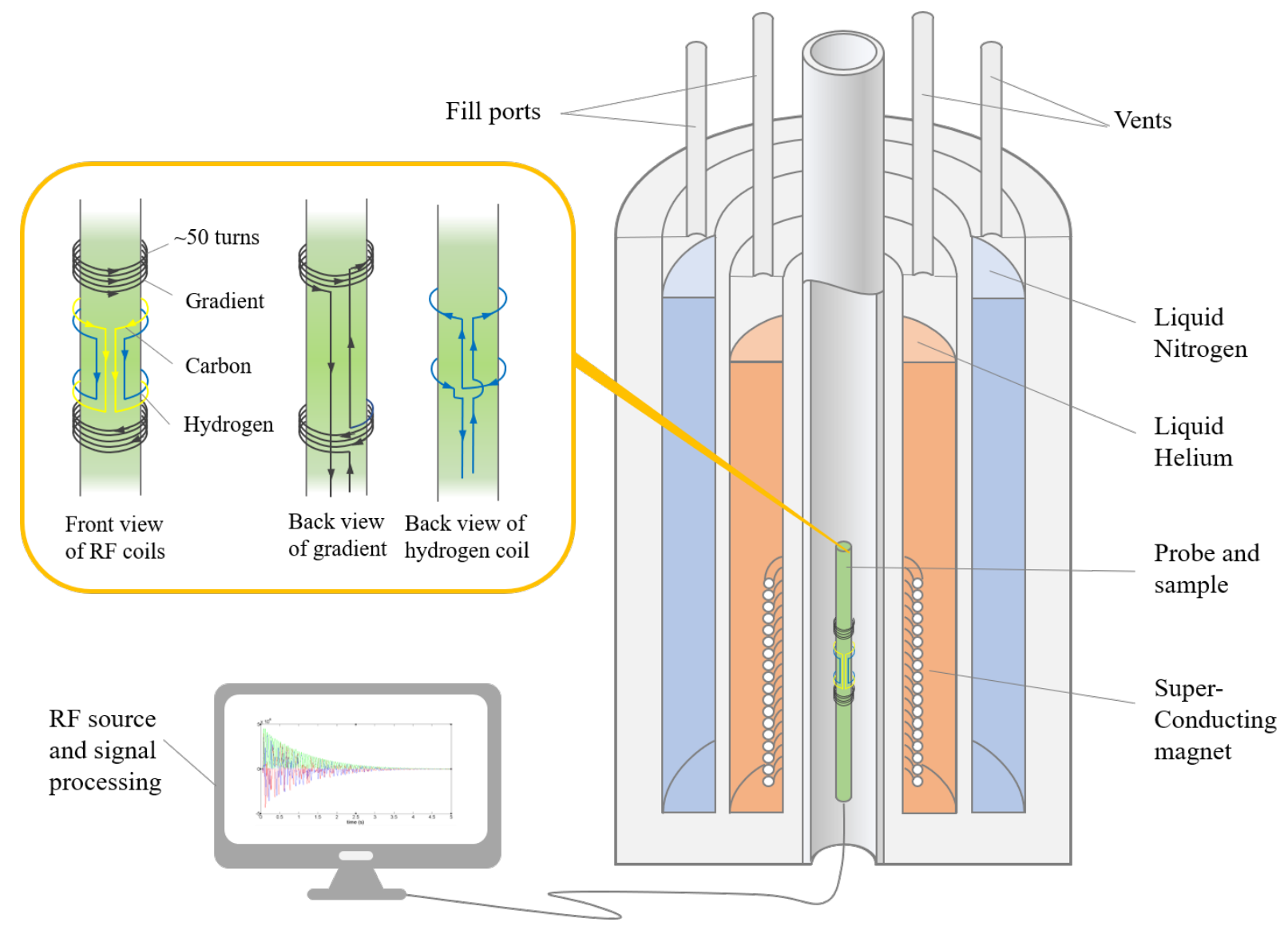

Figure 2.2: Schematic of a standard NMR system. The magnet and the console are main parts of the whole system. The magnet is superconducting magnet and console has the electronics devices to control the spectrometer. The liquid sample is inserted into the central of the magnet, where it is surrounded by coils which we call probe. And radio frequency $(\mathrm{RF})$ pulse can be applied in the probe to control the spins. 


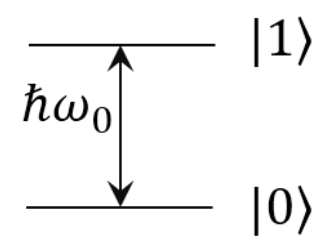

Figure 2.3: Energy diagram of a single spin-1/2.

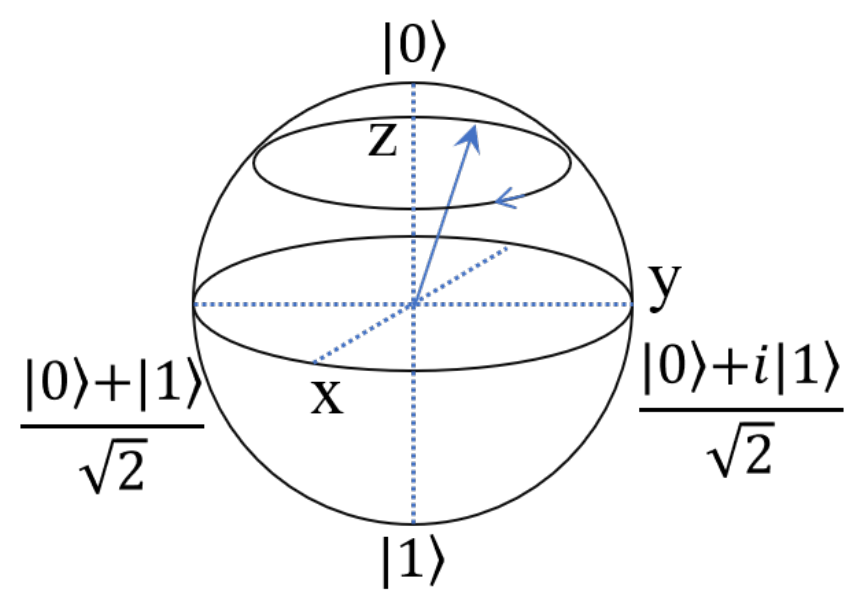

Figure 2.4: Precession of a spin-1/2 particle in a static magnetic field. 


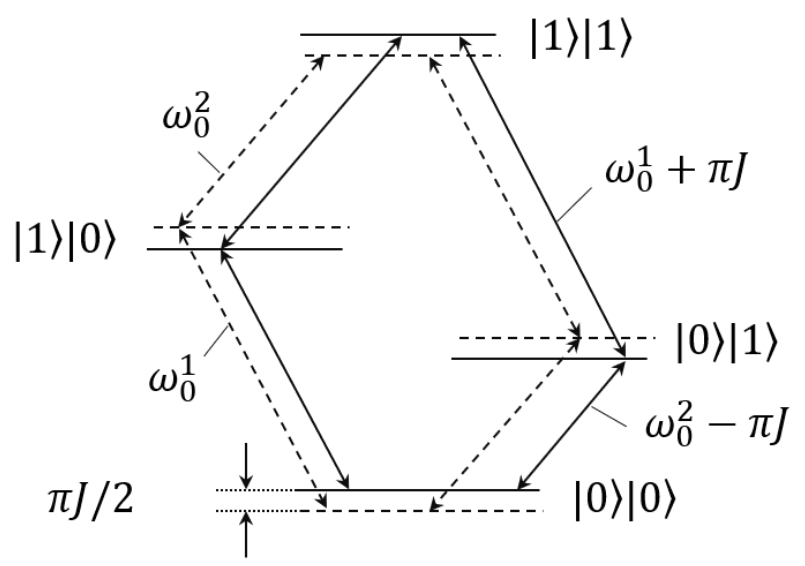

Figure 2.5: Energy level diagram. dashed lines for two uncoupled spins, and solid lines for two spins coupled by a Hamiltonian $H_{J}=\hbar \sum_{i<j}^{n} 2 \pi J_{i j} I_{z}^{i} I_{z}^{j}$. 


\section{Chapter 3}

\section{Scheme for eliminating the synthesizer phase noise}

In this chapter, Ramsey experiment will be firstly reviewed. Then, the effect of phase noise in Ramsey experiment will be presented. To suppress synthesizer phase noise in Ramsey experiment, we proposed two methods in this thesis, which use two antennas for control and detection and one antenna for control and detection, respectively. The proposal uses two single-spin systems in opposite static magnetic fields, and the two spins can experience the phase noise in opposite ways. Therefore, the phase noise can be either canceled or separated from the effect of the environmental field. Finally, Hahn echo experiment and stimulated echo experiment will be briefly reviewed. And our proposed methods can be extended to the Hahn echo and the stimulated echo sequences.

\subsection{Ramsey experiment}

The double-pulse experiment shown in Figure. 3.1 is known as a Ramsey interference experiment [76]. In NMR, there are two $\pi / 2$ pulse and a time delay $t$ in the middle of two pulses to form a Ramsey experiment. Normally Ramsey experiment is described in the rotating frame of radio frequency. The first $\pi / 2$ pulse rotates the qubit into $x-y$ plane, then precession of qubit about $\hat{z}$ for time $t$. Finally the second $\pi / 2$ pulse rotates the qubit back to $\hat{z}$.

For single qubit, the final state will not depend on time $t$, if the qubit is on resonance with the RF field. If the RF field and the qubit precession are not on resonance with offset $\delta \omega$, a beating pattern will display as a function of $\delta \omega t$, which is called Ramsey fringes. 


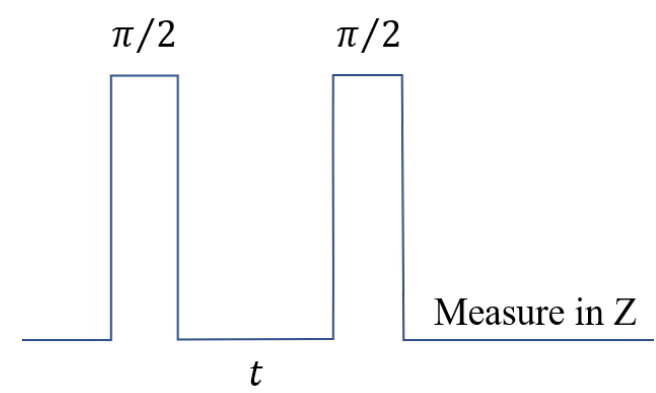

Figure 3.1: Ramsey sequence contains two $\pi / 2$ pulses the time $t$ are same value.

For coupled qubits, the information of coupling strengths can be analyzed if we have the beating pattern which is the basis for the two-dimensional correlation spectroscopy.

\subsection{Synthesizer noise in Ramsey experiment}

In section 3.1 we introduced Ramsey experiment. In this section, the phase noise will be counted in Ramsey experiment. We will take a close look at how the synthesizer phase noise affects the Ramsey experiment.

The internal Hamiltonian and the control Hamiltonian of a single spin-half system in a static magnetic field with strength $B_{0}$ can be written as

$$
\begin{aligned}
H_{\text {in }} & =\left(\omega_{0}+\omega_{\alpha}\right) \frac{\sigma_{z}}{2}, \\
H_{\text {con }} & =\Omega \cos \left(\omega_{0} t+\phi_{\text {con }}+\phi(t)\right) \frac{\sigma_{x}}{2} .
\end{aligned}
$$

where $\omega_{0}$ is the spin's Zeeman frequency in a magnetic field $B_{0}$, environmental field $B_{\alpha}$ will induce an equivalent frequency which is denoted as $\omega_{\alpha}$, which is to be detected. $\Omega$ is the amplitude of the control microwave pulse. $\phi_{c o n}+\phi(t)$ is the phase of the control pulse, where $\phi_{c o n}$ is a well-controlled term and $\phi(t)$ is the synthesizer phase noise term.

Equation (3.1) can be simplified in a rotating frame. As the coherence of a spin is usually measured relative to the reference frame provided by the synthesizer, it is convenient to choose the synthesizer frequency, $\omega_{0}+\omega_{n}(t)$ with $\omega_{n}(t) \equiv \dot{\phi}(t)$, as the frequency of the 
rotating frame to work in. In this rotating frame and after rotating wave approximation, Eq. (3.1) can be expressed as,

$$
\begin{aligned}
\tilde{H}_{i n} & =\left(\omega_{\alpha}-\omega_{n}\right) \frac{\sigma_{z}}{2} \\
\tilde{H}_{c o n} & =\Omega\left(\cos \left(\phi_{c o n}\right) \frac{\sigma_{x}}{2}+\sin \left(\phi_{c o n}\right) \frac{\sigma_{y}}{2}\right) .
\end{aligned}
$$

The above equations indicate that the impacts on the spin dynamics from the environmental field $B_{\alpha}$ and the synthesizer phase noise have equal weight. So it is hard to separate $\omega_{\alpha}$ from $\omega_{n}$.

Next, we discuss what happens if the Ramsey sequence is used to detect environmental field $B_{\alpha}$. The Ramsey experiment is widely used in quantum metrology. A Ramsey sequence usually contains two $\pi / 2$ rotation pulses (see Figure. 3.2). A spin polarization along $z$-axis can be rotated to the $x y$-plane of the Bloch sphere by the first pulse, where the spin precesses if a frequency offset in the rotating frame exists. After a period, a phase $\theta$ is accumulated in the $x y$-plane, and a second pulse rotates the spin back to the $z$ direction for detection. The amount of the polarization that is restored back to $z$-axis is proportional to $\cos (\theta)$ if the two pulses have the same phase. Therefore, by detecting the $z$ direction polarization, $\theta$ can be derived and hence the offset frequency. If this offset frequency is caused by an environmental field $B_{\alpha}$, then $B_{\alpha}$ can be detected this way. However, from Eq. (3.2), the synthesizer phase noise causes an additional unwanted frequency offset, $-\omega_{n}$. The Ramsey experiment detects the phase accumulated by the offset frequency $\omega_{\alpha}-\omega_{n}$ instead of $\omega_{\alpha}$. Therefore, the synthesizer noise can cause an error in the measurement of the environmental field strength.

\subsection{Methods to suppress synthesizer phase noise}

In this section, we proposed two methods to suppress synthesizer phase noise. They are two-antenna method and one-antenna method. In the following, the methods will be introduced in detail.

The key of the scheme stated here is an observation that the control pulse as in Eq. (3.1) has both components that co-rotates and counter-rotates with the spin system:

$$
\begin{aligned}
H_{\text {con }} & =\Omega \cos \left(\omega_{0} t+\phi_{\text {con }}+\phi(t)\right) \frac{\sigma_{x}}{2} \\
& =\Omega\left[\exp \left(i\left(\omega_{0} t+\phi_{\text {con }}+\phi(t)\right)\right)+\exp \left(-i\left(\omega_{0} t+\phi_{\text {con }}+\phi(t)\right)\right)\right] \frac{\sigma_{x}}{4},
\end{aligned}
$$




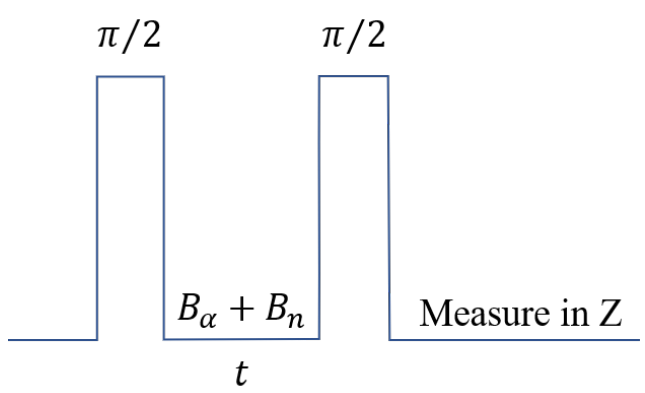

Figure 3.2: The two-pulse Ramsey sequence. It can be used to detect an offset magnetic field $B_{\alpha}$ in the environment. However, the synthesizer phase noise effectively adds a noise term $B_{n}$. In a Ramsey experiment, the initial state is the thermal equilibrium state $\sigma_{z}$ and the final state after the second $\pi / 2$ rotation is $\cos \left(\theta_{\alpha}-\theta_{n}\right) \sigma_{z}+\sin \left(\theta_{\alpha}-\theta_{n}\right) \sigma_{y}$ (the two rotations are assumed to be along $y$-axis). The second $\pi / 2$ rotation is used to restore the spin polarization to $z$-axis for detection. Here $\theta_{\alpha}$ and $\theta_{n}$ are the phases induced by $B_{\alpha}$ and the synthesizer noise, respectively. These two phases acquired by the spin are difficult to separate.

where $i$ is the unit imaginary number. When a spin system has Zeeman frequency $\omega_{0}$, the co-rotating term $\exp \left(-i\left(\omega_{0} t+\phi_{c o n}+\phi(t)\right)\right)$ is used for controlling it. Because of rotating wave approximation, the $\exp \left(i\left(\omega_{0} t+\phi_{c o n}+\phi(t)\right)\right)$ term can be neglected. If there exists a spin system with Zeeman frequency $-\omega_{0}$, it can be controlled by $H_{c o n}$ due to the $\exp \left(i\left(\omega_{0} t+\phi_{\text {con }}+\phi(t)\right)\right)$ term.

In the following, we show that the synthesizer phase noise affects such two systems in opposite ways, which can be used to eliminate the noise effect.

For simplicity, we neglect the second pulse in the Ramsey sequence and assume the $x y$-polarization detection (see Figure. 3.3).

Here, we discuss two different configurations, which utilize two antennas (Figure. 3.4(a)) and one antenna (Figure. 3.4(b)), respectively. Suppose there are two single-spin systems in two static magnetic fields with opposite directions and they are exposed to the same environmental field $B_{\alpha}$ (see Figure. 3.4).

In the two-antenna configuration, the two spins are controlled and detected by the two antennas separately, while by a single antenna in the one-antenna configuration. It should be mentioned that the two-antenna configuration can be applied with different detection techniques. Although $x y$-plane measurement is mostly used in magnetic resonance systems, 


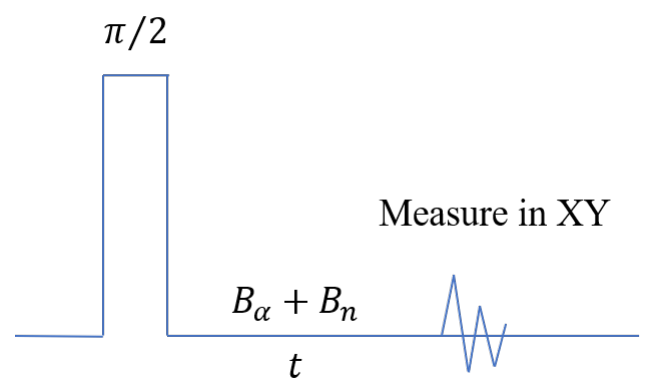

Figure 3.3: Ramsey sequence when spin polarizations in $x y$-plane can be detected. The second $\pi / 2$ rotation as in Figure. 3.2 can be neglected.

similar conclusions can be obtained for other systems with $z$ direction measurement. However, as will be discussed below, the one-antenna configuration needs quadrature detection and thus is less general and more restricted to magnetic resonance systems.

In both the two-antenna and one-antenna configurations, the two spins are controlled using the same synthesizer source with the control Hamiltonian in Eq. (3.3). The internal Hamiltonians for the spin in $B_{0}$ (system 1) and the spin in $-B_{0}$ (system 2) are $H_{1}=$ $\left(\omega_{0}+\omega_{\alpha}\right) \frac{\sigma_{z}}{2}$ and $H_{2}=\left(-\omega_{0}+\omega_{\alpha}\right) \frac{\sigma_{z}}{2}$, respectively. With the rotating wave approximation, the $\exp \left(-i\left(\omega_{0} t+\phi_{\text {con }}+\phi(t)\right)\right) \frac{\sigma_{x}}{2}$ and $\exp \left(i\left(\omega_{0} t+\phi_{\text {con }}+\phi(t)\right)\right) \frac{\sigma_{x}}{2}$ terms in $H_{\text {con }}$ are used to control system 1 and system 2 separately.

\subsubsection{Two-antenna method}

First, we analyse the dynamics of the two systems in the two-antenna configuration.

For system 1 , in the rotating frame, $U_{1}=\exp \left(-i\left(\omega_{0}+\omega_{n}\right) t \frac{\sigma_{z}}{2}\right)$, the effective total Hamiltonian is

$$
\begin{aligned}
\tilde{H}_{1}^{t o t} & =U_{1}^{\dagger}\left(H_{1}+H_{c o n}\right) U_{1}-\left(\omega_{0}+\omega_{n}\right) \frac{\sigma_{z}}{2} \\
& \approx\left(-\omega_{n}+\omega_{\alpha}\right) \frac{\sigma_{z}}{2}+\Omega\left(\cos \left(\phi_{c o n}\right) \frac{\sigma_{x}}{2}+\sin \left(\phi_{c o n}\right) \frac{\sigma_{y}}{2}\right) .
\end{aligned}
$$

The $\pi / 2$ rotation pulse can be expressed as $R_{1}(\pi / 2)=\exp \left(-i \frac{\pi}{4} \sigma\right)$, where $\sigma=\cos \left(\phi_{\text {con }}\right) \sigma_{x}+$ $\sin \left(\phi_{\text {con }}\right) \sigma_{y}$. 
Therefore, in a Ramsey experiment, the state evolution is as follows: the spin starts in the thermal equilibrium state $\sigma_{z}$; after $R_{1}(\pi / 2)$, the state is $\sin \left(\phi_{\text {con }}\right) \sigma_{x}-\cos \left(\phi_{\text {con }}\right) \sigma_{y}$; after a free evolution the state becomes

$$
\rho_{1}^{t}=\sin \left(\phi_{\text {con }}+\theta_{1}\right) \sigma_{x}-\cos \left(\phi_{\text {con }}+\theta_{1}\right) \sigma_{y},
$$

where $\theta_{1}=\int\left(-\omega_{n}+\omega_{\alpha}\right) d t=-\theta_{n}+\theta_{\alpha}$. As expected, both $B_{\alpha}$ and the synthesizer phase noise contribute to the accumulated phase $\theta_{1}$ during the free evolution.

For system 2, in the rotating frame, $U_{2}=\exp \left(i\left(\omega_{0}+\omega_{n}\right) t \frac{\sigma_{z}}{2}\right)$, the effective total Hamiltonian is

$$
\begin{aligned}
\tilde{H}_{2}^{\text {tot }} & =U_{2}^{\dagger}\left(H_{2}+H_{c o n}\right) U_{2}+\left(\omega_{0}+\omega_{n}\right) \frac{\sigma_{z}}{2} \\
& \approx\left(\omega_{n}+\omega_{\alpha}\right) \frac{\sigma_{z}}{2}+\Omega\left(\cos \left(\phi_{c o n}\right) \frac{\sigma_{x}}{2}-\sin \left(\phi_{c o n}\right) \frac{\sigma_{y}}{2}\right) .
\end{aligned}
$$

The $\pi / 2$ rotation pulse can be expressed as $R_{2}(\pi / 2)=\exp \left(-i * \frac{\pi}{4} \sigma\right)$, where $\sigma=\cos \left(\phi_{\text {con }}\right) \sigma_{x}-$ $\sin \left(\phi_{\text {con }}\right) \sigma_{y}$. Comparing $\tilde{H}_{2}^{\text {tot }}$ with $\tilde{H}_{1}^{\text {tot }}$, one major difference is the sign in front of $\omega_{n}$.

We can expect a different effect of the phase noise here. The state evolves as follows: the spin starts in its thermal equilibrium state $-\sigma_{z}$; after $R_{2}(\pi / 2)$, the state is $\sin \left(\phi_{\text {con }}\right) \sigma_{x}+$ $\cos \left(\phi_{\text {con }}\right) \sigma_{y}$; after a free evolution of a duration $t$ the state becomes

$$
\rho_{2}^{t}=\sin \left(\phi_{c o n}-\theta_{2}\right) \sigma_{x}+\cos \left(\phi_{c o n}-\theta_{2}\right) \sigma_{y},
$$

where $\theta_{2}=\int\left(\omega_{n}+\omega_{\alpha}\right) d t=\theta_{n}+\theta_{\alpha}$.

As the $x y$-plane polarization can be detected, phases $\theta_{1}$ and $\theta_{2}$ in states $\rho_{1}^{t}$ and $\rho_{2}^{t}$ can be easily measured in their rotating frames separately. By averaging $\theta_{1}$ and $\theta_{2}$, the phase accumulated due to the phase noise, $\theta_{n}$, is canceled, while the phase induced by $B_{\alpha}, \theta_{\alpha}$, remains. Therefore, $B_{\alpha}$ can be measured accurately.

In the above analysis, we assume the two systems are controlled using the same synthesizer source but the detection of the two systems happens separately in their own rotating frames. In other words, the two systems are controlled and detected by two separate antennas, which are connected to the same synthesizer (see Figure. 3.4(a)). In this configuration, components and lines are needed for separating the synthesizer signal and connecting to the two antennas. Therefore, special attention should be paid to the symmetric design to avoid inducing an additional difference between the two systems. 

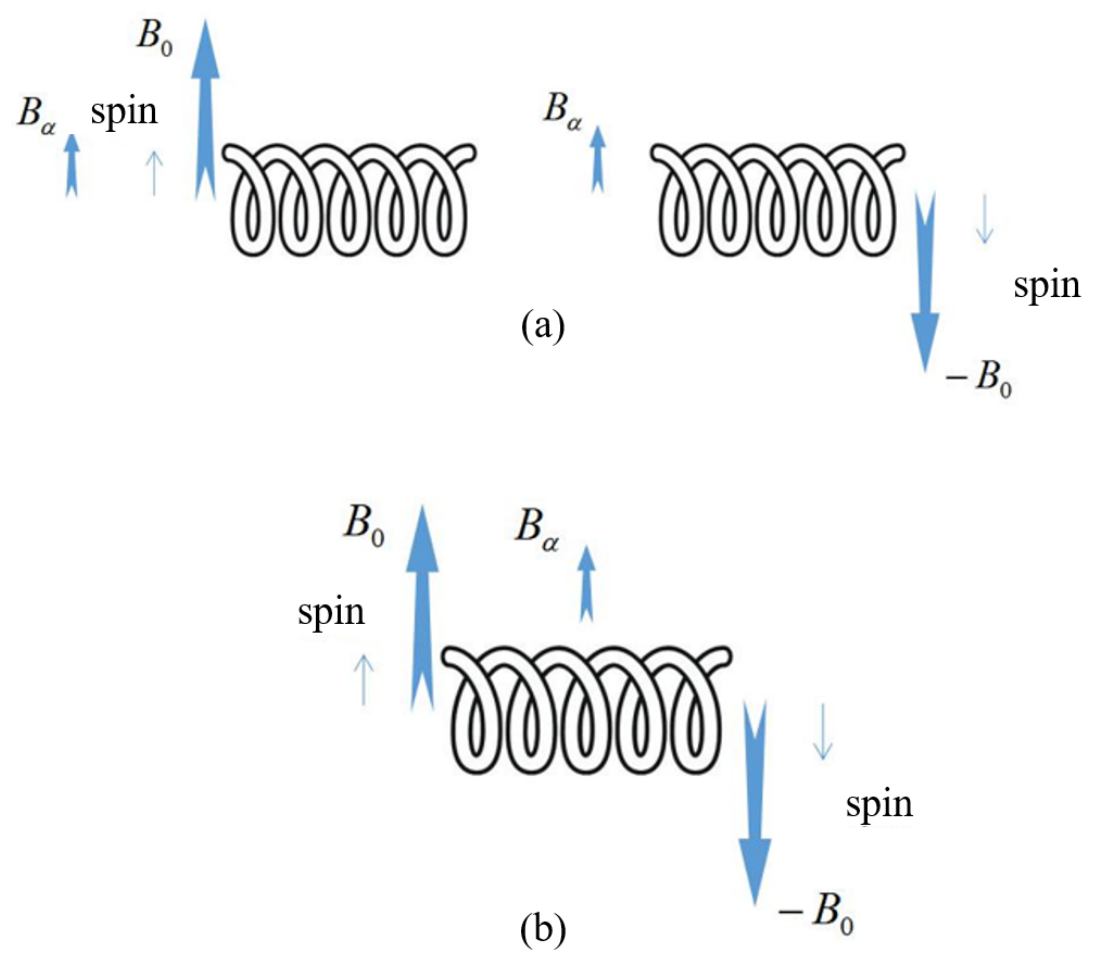

Figure 3.4: The two-antenna configuration (a), and one-antenna configuration (b) of the proposed scheme. Here multi-round coils are used to represent antennas. Two single-spin systems are placed in two opposite static magnetic fields, $B_{0}$ and $-B_{0}$. They are controlled using the same synthesizer and exposed to the same environment offset field $B_{\alpha}$ which is to be sensed. 


\subsubsection{One-antenna method}

In the one-antenna configuration (Figure. 3.4(b)), the control of the two systems is the same as in the two-antenna configuration, while the detection is different. The single antenna detects the sum of the signals from the two spins. Therefore, we cannot treat the signals of the two spins in their rotating frames separately during detection. Both states $\rho_{1}^{t}$ and $\rho_{2}^{t}$ should be transferred back to the lab frame and summed up. In the lab frame,

$$
\begin{aligned}
& \rho_{1}^{t} \rightarrow \rho_{1, L}^{t}=\sin \left(\phi_{\text {con }}+\theta_{\alpha}+\omega_{o} t\right) \sigma_{x}-\cos \left(\phi_{\text {con }}+\theta_{\alpha}+\omega_{o} t\right) \sigma_{y}, \\
& \rho_{2}^{t} \rightarrow \rho_{2, L}^{t}=\sin \left(\phi_{\text {con }}-\theta_{\alpha}+\omega_{o} t\right) \sigma_{x}+\cos \left(\phi_{\text {con }}-\theta_{\alpha}+\omega_{o} t\right) \sigma_{y} .
\end{aligned}
$$

It is not surprising that the phase noise term $\theta_{n}$ does not appear in states $\rho_{1, L}^{t}$ and $\rho_{2, L}^{t}$, as the phase noise is associated with the reference rotating frames which are provided by the synthesizer. Assuming the single antenna detects the $x$-polarization, the detected signal $S_{x}$ is

$$
S_{x}=A\left(\sin \left(\phi_{c o n}+\theta_{\alpha}+\omega_{0} t\right)+\sin \left(\phi_{c o n}-\theta_{\alpha}+\omega_{0} t\right)\right)=2 A \sin \left(\phi_{c o n}+\omega_{0} t\right) \cos \left(\theta_{\alpha}\right) .
$$

Here, $A$ is an amplitude determined by the signal receiver and other hardware components. Without loss of generality, we can assume $A=1$.

In realistic conditions, collecting high-frequency signals is demanding. Therefore, a signal such as in Eq. (3.9), before recording, needs to be mixed with the synthesizer signal for demodulation to get a signal with a lower frequency. Demodulation includes

multiplication of the detected signal with the synthesizer signal followed by a low pass filter.

In NMR systems usually quadrature detection is used: the spin signal is split into two paths where 0 and $\pi / 2$ phase-shifted synthesizer signals are multiplied. In a case with a single spin frequency in an antenna, the two signals generated by the two multiplications, after a low pass filter, corresponding to the $x$ and $y$ polarizations (or real and imaginary parts) of the spin in its rotating frame, respectively. This is how detection can be done in the two-antenna configuration case in NMR systems. However, in the one-antenna configuration, we have $\omega_{0}$ and $-\omega_{0}$ spin frequencies in a single antenna, thus the two signals from quadrature detection do not correspond to the $x$ or $y$ polarizations of either the spins in their rotating frames. But we keep using 'real' and 'imaginary' to call the two signals. As analyzed above, demodulation of $S_{x}$ is as follows: 
(1) Real part of the signal:

$$
\begin{aligned}
S_{x} * \cos \left(\omega_{0} t+\theta_{n}\right) & =2 \sin \left(\phi_{\text {con }}+\omega_{o} t\right) \cos \left(\theta_{\alpha}\right) \cos \left(\omega_{0} t+\theta_{n}\right) \\
& =\cos \left(\theta_{\alpha}\right)\left[\sin \left(\phi_{c o n}+2 \omega_{0} t+\theta_{n}\right)+\sin \left(\phi_{\text {con }}-\theta_{n}\right)\right] \\
& \stackrel{\text { low pass filter }}{\longrightarrow} \cos \left(\theta_{\alpha}\right) \sin \left(\phi_{\text {con }}-\theta_{n}\right) .
\end{aligned}
$$

(2) Imaginary part of the signal:

$$
\begin{aligned}
S_{x} * \sin \left(\omega_{0} t+\theta_{n}\right) & =2 \sin \left(\phi_{\text {con }}+\omega_{o} t\right) \cos \left(\theta_{\alpha}\right) \sin \left(\omega_{0} t+\theta_{n}\right) \\
& =-\cos \left(\theta_{\alpha}\right)\left[\cos \left(\phi_{\text {con }}+2 \omega_{0} t+\theta_{n}\right)-\cos \left(\phi_{\text {con }}-\theta_{n}\right)\right] \\
& \stackrel{\text { low pass filter }}{\longrightarrow} \cos \left(\theta_{\alpha}\right) \cos \left(\phi_{\text {con }}-\theta_{n}\right) .
\end{aligned}
$$

From Eqs. (3.10) and (3.11), we can see that demodulation introduces the phase noise again. Although the noise term $\theta_{n}$ exists in the final collected signals, different from the case with only one spin system, the environment information $\theta_{\alpha}$ can be derived from the amplitude instead of the phase of the signals and thus will not be affected by the synthesizer phase noise.

As analyzed above, both in the two-antenna and one-antenna configurations, the noise term $\theta_{n}$ can be removed from the measurement of the environment term $\theta_{\alpha}$ and thus the sensing accuracy can be improved. Besides, the application of one-antenna configuration is general. Although thermal equilibrium states are used as the initial states in the analysis, if

the two spins are initialized in their ground states, the conclusion remains the same. And if the detection is along $z$-direction, the only change needed for the one-antenna configuration to work is to include the second $\pi / 2$ pulse in the Ramsey sequence.

\subsection{Hahn echo sequence and stimulated echo sequence}

In this section, Hahn echo sequence and stimulated echo sequence will be introduced and how to use methods proposed in section 3.3 will be discussed.

\subsubsection{Hahn echo sequence}

In 1950 Ewrin Hahn first detected the echoes in NMR system. As shown in Figure.3.5, Hahn echo sequence contains two pulses the first pulse is a $\pi / 2$ rotation pulse and the 
second pulse was also $\pi / 2$ rotation pulse in the first paper of Hahn, and it was further developed by Carr and Purcell who changed the $\pi / 2$ pulse to a $\pi$ pulse. The first $\pi / 2$ is used to rotate the state into $x-y$ plane from $\hat{z}$ axis. After a time $t$ evolution the signal will decay because of the inhomogeneities of the magnetic field. The second pulse $\pi$ is applied to flip the spins. And during another time $t$ the spins refocusing and an echo can be observed at time $2 t$.

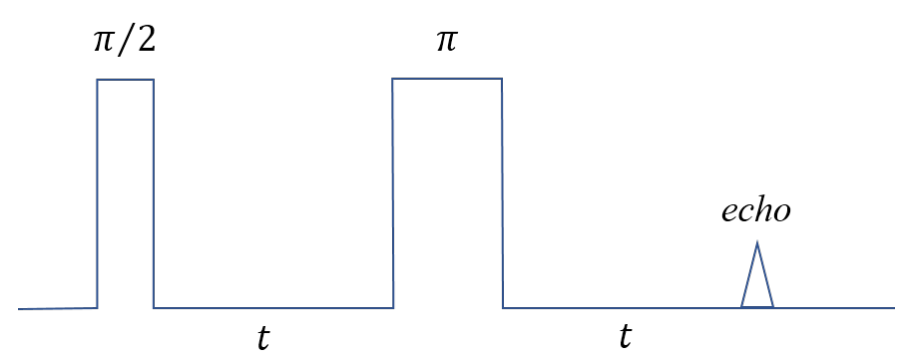

Figure 3.5: Hahn echo is similar to Ramsey sequence. But the second $\pi / 2$ pulse is replaced by a $\pi$ pulse, so Hahn echo contains one $\pi / 2$ pulse and one refocusing $\pi$ pulse.

\subsubsection{Stimulated echo sequence}

A basic pulse sequence used to generate an stimulated echo (STE) sequence is shown in Figure.3.6. Note the terminology switch to $t$ for the first and third intervals and $T$ for the middle interval. The combination of the first and third intervals is referred to as the echo time because this is the time that the spins are in the $x-y$ plane. Spin in $x$ direction will continue to refocus to form the spin echo. The third $\pi / 2$ pulse will flip the magnetization vector to $y$ direction after time $t$ there will be an STE along $-y$.

The first $\pi / 2$ pulse rotates the spin into $x-y$ plane, and after time $t$ spins dephase in the $x-y$ plane totally. The second $\pi / 2$ pulse does not affect the spin along $x$ direction, it will flip spin in $y$ direction into $z$ direction.

A magnetic field gradient in the first interval is used to ensure dephasing of the spins after the first pulse. This dephasing must be refocused with a magnetic field gradient in the third interval. Note the orientation of the gradient in the third interval; it is in the same direction as the first interval because the direction of the spins has been reversed by the rf pulses (see Figure. 2). Although a constant magnetic field gradient (a poor shim) 


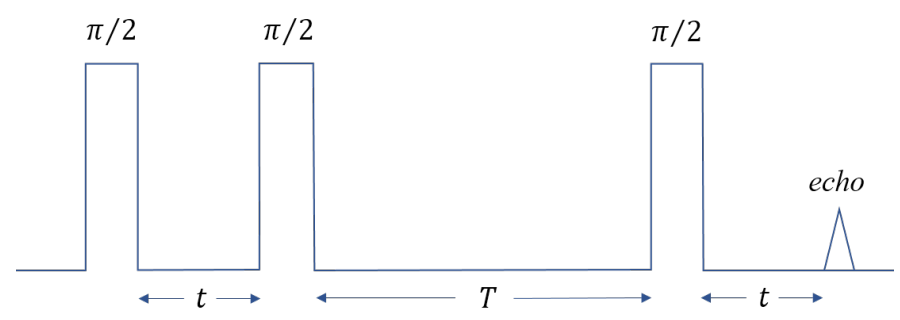

Figure 3.6: Stimulated echo contains three $\pi / 2$ pulse, $t$ and $T$ are different value.

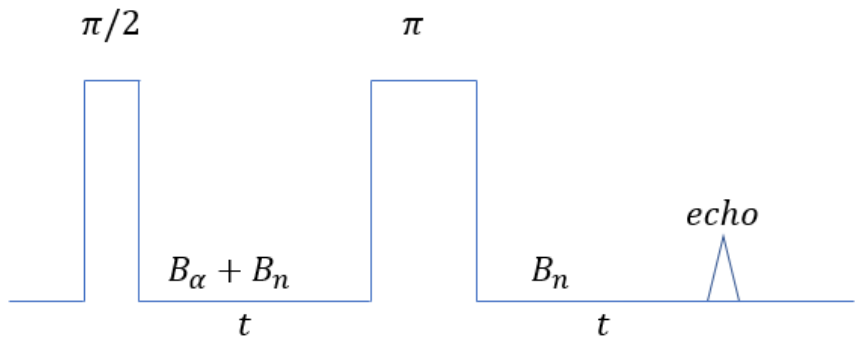

Figure 3.7: Hahn echo sequence. The initial state of a Hahn echo experiment is usually the thermal equilibrium state. Here, we assume the environment offset field $B_{\alpha}$ is not present after the $\pi$ refocusing pulse.

also would be effective in dephasing the spins during t, it also will lead to corresponding effects of magnetic field inhomogeneity in the echo and hence in the spectrum or image.

The magnetic field gradient in the second interval will eliminate the formation of the first spin echo, and hence also the second spin echo. (The first spin echo will form, however, in the specific case where the gradient in the middle interval is put before the time of the formation of the spin echo and matches a gradient that might be present in the first interval.) Similarly, the magnetic field gradient in the third interval will eliminate the formation of the third spin echo, because the spins associated with this echo have not experienced the first magnetic field gradient and therefore will not refocus.

\subsubsection{Extending to Hahn echo and Stimulated echo sequence}

Sometimes the Hahn echo sequence (Figure. 3.7) and the stimulated echo sequence (Figure. 3.8) are needed in a sensing process, for example, to cancel a static field inhomogeneity. 


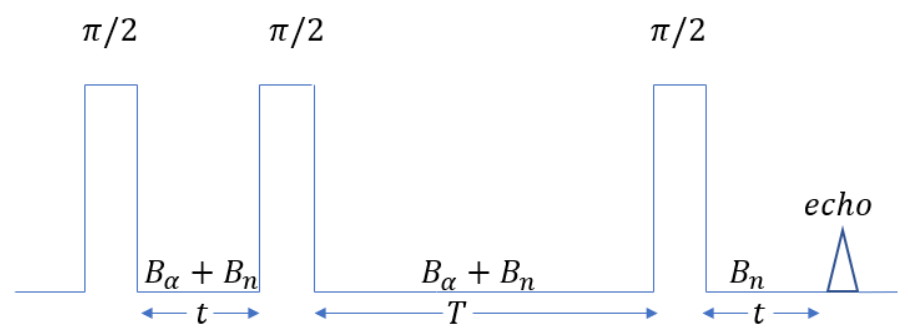

Figure 3.8: Stimulated echo sequence. The initial state of a stimulated echo experiment is usually the thermal equilibrium state. Here, we assume the environment offset field $B_{\alpha}$ is not present after the third $\pi / 2$ pulse.

It can be proven that the scheme discussed above in Ramsey experiments can be easily extended to the two experiments. However, it should be mentioned that the environmental field $B_{\alpha}$ should not appear during the whole sequence for either the Hahn echo or stimulated echo experiments.

In the Hahn echo experiment, if $B_{\alpha}$ exists both before and after the $\pi$ refocusing pulse, part of the accumulated phase induced by $B_{\alpha}$ can be canceled by the refocusing pulse. In the extreme case where $B_{\alpha}$ is constant, the phase $\theta_{\alpha}$ will be 0 at the time the echo forms.

In the stimulated echo experiment, the phase noise and the environmental field between the second and third pulses do not affect the spin signals as the spin polarization is mainly stored along $z$ direction between the two pulses. Similar to the Hahn echo experiment, if $B_{\alpha}$ exists after the third pulse, the accumulated phases from $B_{\alpha}$ 's effect before the second pulse and after the third pulse can cancel partly. If $B_{\alpha}$ is constant, the phase $\theta_{\alpha}$ is also zero when the echo forms.

Therefore, we conclude that, for good sensitivity, it is better to avoid $B_{\alpha}$ after the refocusing pulse in a Hahn echo experiment and after the third pulse in a stimulated echo experiment (see Figures. 3.7 and 3.8). Under such an assumption, the analysis of the proposed scheme in the two experiments is exactly the same as in the Ramsey experiment. With the two-coil configuration, the phase noise can be canceled completely. With the onecoil configuration, the effect of $B_{\alpha}$ is encoded in the signal amplitude and thus separated from the synthesizer phase noise. 


\section{Chapter 4}

\section{Experiment}

In this chapter, experiments on NMR system will be introduced in detail. First, the preparation of the experiment in NMR system is introduced. Then, the data analysis of two sets of experiments is discussed. In detail, the proposed one-antenna configuration of the Ramsey experiment using the two-spin NMR system and one-spin NMR system is analyzed.

\subsection{Experiment preparation}

We demonstrated the proposed one-antenna configuration of the Ramsey experiment using a two-spin NMR system, ${ }^{13} \mathrm{C}$ labeled Chloroform, on a $400 \mathrm{MHz}$ Bruker spectrometer. The molecule structure and Hamiltonian parameters are illustrated in Figure. 4.1.

In our real NMR experiment, we use the energy splitting by the $J$-coupling between ${ }^{13} \mathrm{C}$ and ${ }^{1} \mathrm{H}$ to simulate the energy difference caused by the two opposite static magnetic fields required by the scheme. In other words, the two frequencies of ${ }^{1} \mathrm{H}$ separated by the $J$-coupling $215.2 \mathrm{~Hz}$ are used to simulate the two frequencies of two spins in opposite magnetic fields $B_{0}$ and $-B_{0}$ with $\omega_{0}=107.6 \mathrm{~Hz}$.

We collect the free induction decay (FID) data in ${ }^{1} \mathrm{H}$ 's rotating frame, which contains the $\omega_{0}$ and $-\omega_{0}$ frequencies. Therefore, the ${ }^{1} \mathrm{H}$ 's rotating frame is used to simulate the lab frame in the proposed scheme.

A perfect sinusoidal signal with the form $\cos \left(\omega_{0} t\right)$ is generated numerically to act as a perfect synthesizer signal. 


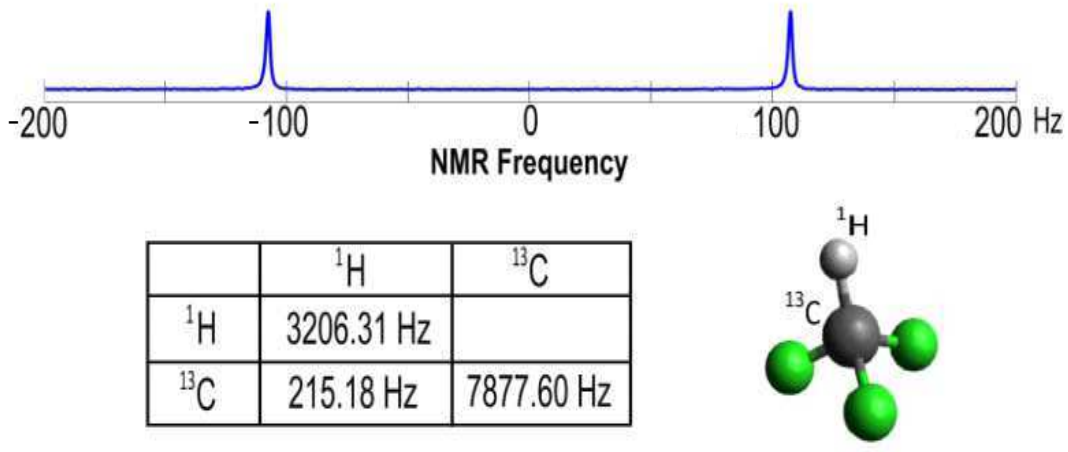

Figure 4.1: Chloroform molecule structure. The ${ }^{13} \mathrm{C}$ and ${ }^{1} \mathrm{H}$ are spin-half nuclei. Their chemical shifts and $J$-coupling constant are listed as the diagonal and off-diagonal elements in the table. The topmost panel shows a thermal spectrum of ${ }^{1} \mathrm{H}$, where the two peaks associate with the two Zeeman eigenstates, spin-up and spin-down, of ${ }^{13} \mathrm{C}$ in a magnetic field.

Random phase noise is injected to a perfect cosine signal to simulate the synthesizer signal with phase noise. Random Gaussian noise is used here, which is generated using the method discussed in [77]. The noise power spectrum used for noise generation is shown in Figure.4.2. The generated synthesizer signal is used for demodulating the collected FID signals.

\subsection{Data analysis}

In the following, two sets of experiments using chloroform will be introduced and the data analysis will be discussed. The first set of the experiments is implemented in the absence of the offset field B. And the second set of experiments are implemented in the case that the offset field B exists. In order to demonstrate the advantage of the proposed two-spin scheme in canceling the synthesizer phase noise, each set of experiments includes two experiments: one is simulating a two-spin system in the opposite fields and for comparison, the other is simulating a one-spin system. 


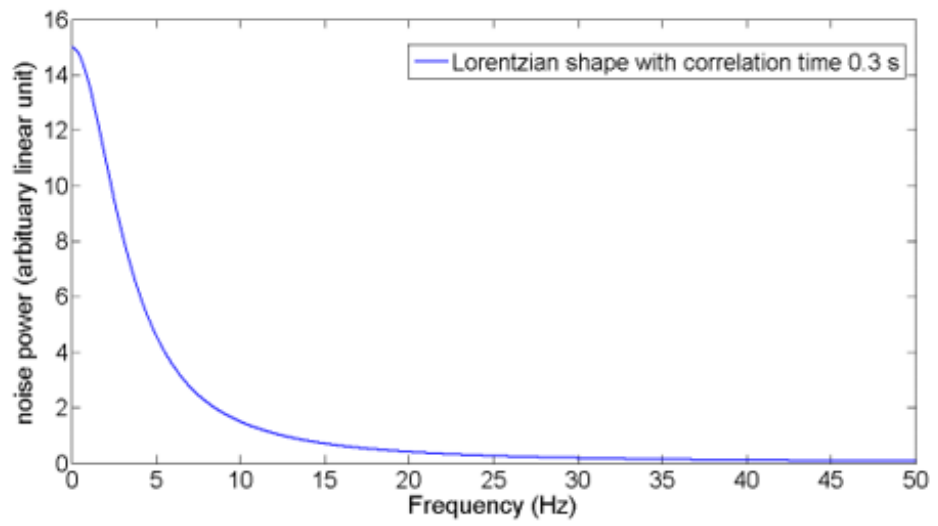

Figure 4.2: The noise power density spectrum used for noise generation.

\subsubsection{Experiments without $B_{\alpha}$}

As mentioned above, the $\mathrm{H}$ rotating frame is used to simulate the lab frame of the proposed scheme. Figure 4.3 shows the FID of the simulated two-spin and one-spin systems in the $\mathrm{H}$ rotating frame. The Fourier transform spectra of the FIDs are shown in Figure. 4.4.

Figure 4.4(a) shows the two peaks of $\pm \omega_{0}$, which are the two frequencies of the simulated two spins in the opposite fields. As a comparison, Figure. 4.4(b) only shows the peak of $\omega_{0}$, which is the frequency of the simulated one-spin system. These two spectra are obtained after the first $\pi / 2$ rotation pulse in the Ramsey experiment. Here we choose $\phi_{\text {con }}=\pi / 2$. To get the second spectrum, the $\pi / 2$ pulse is selective to only the frequency $\omega_{0}$.

Their FID signals after demodulation using the perfect synthesizer signal are shown in Figure.4.5(a) and (b) respectively. It is not strange that the two FID signals have similar shapes, because the detection is chosen to be along $x$ direction where the co-rotating and counter-rotating spins both have the same polarization when there is no offset field $B_{\alpha}$, as can be seen from the coefficients in front of $\sigma_{x}$ in $\rho_{1, L}^{t}$ and $\rho_{2, L}^{t}$ in Eq. (3.8). However, the amplitude of the demodulated FID signal of the two-spin system is twice that of the one-spin system as expected.

When the synthesizer signal has noise, the demodulated FID signals are shown in Figure.4.6. It is not strange that the real and imaginary parts of both experiments are noisy, as the injected noise to the synthesizer signal is phase noise. Figure.4.7 shows the Fourier transform of the FIDs in Figure.4.6. Apart from the major peaks at the zero frequency, there are small peaks from the noise. The major peaks have zero frequencies because of the fact that there is no offset field. The injected noise has a zero statistical mean, which 


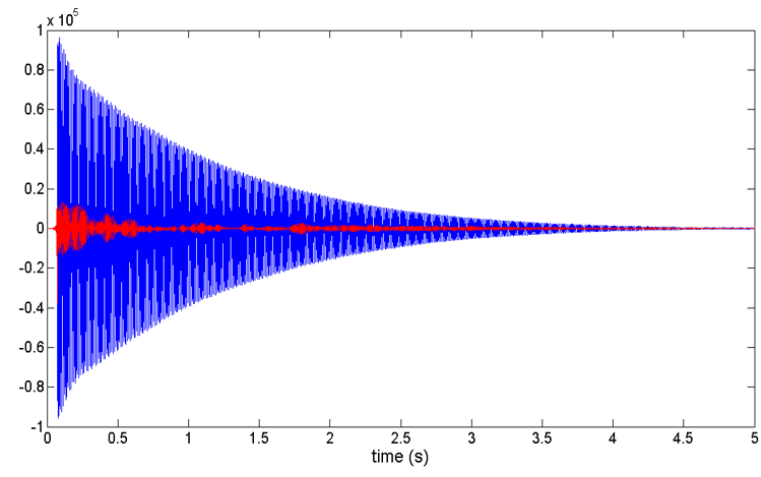

(a) Two-spin

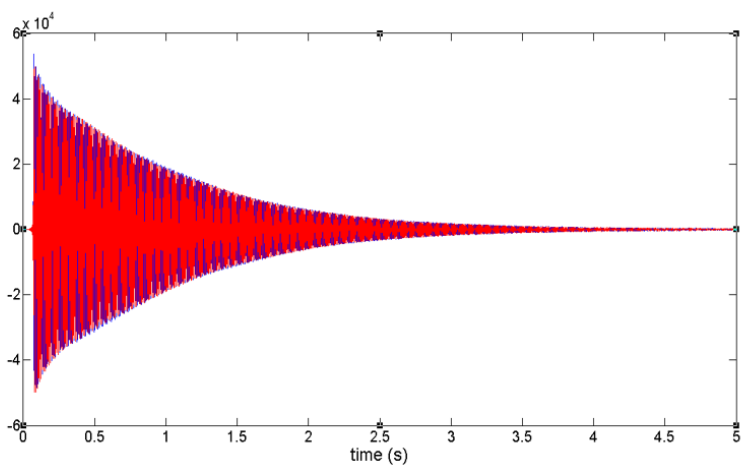

(b) One-spin

Figure 4.3: FID in the $\mathrm{H}$ rotating frame. Blue line represents the real part of FID and the red line is the imaginary part of FID. As shown in the two Figures the real parts are similar but the imaginary part seems cancel out for two-spin system as can be explained by Eq. (3.8).

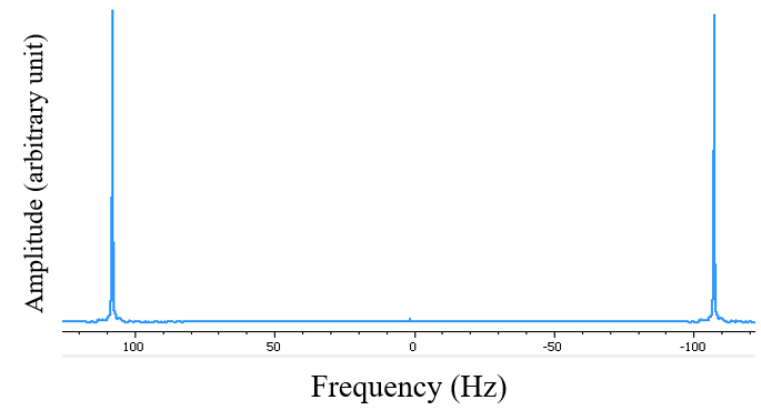

(a) Two-spin

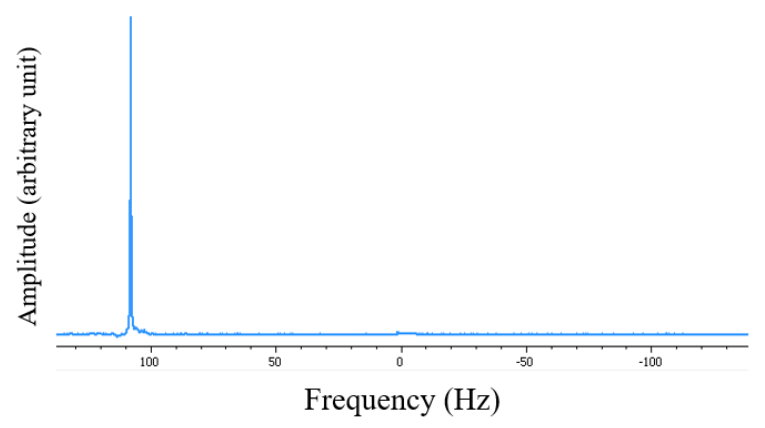

(b) One-spin

Figure 4.4: Experimental frequency spectra. No offset field is present. (a) is the spectrum of the experiment where two spins in two magnetic fields with $\pm \omega_{0}= \pm 107.6 \mathrm{~Hz}$ are simulated. (b) is the spectrum of the experiment where one spin in one magnetic field with $\omega_{0}=107.6 \mathrm{~Hz}$ is simulated for comparison with (a). 


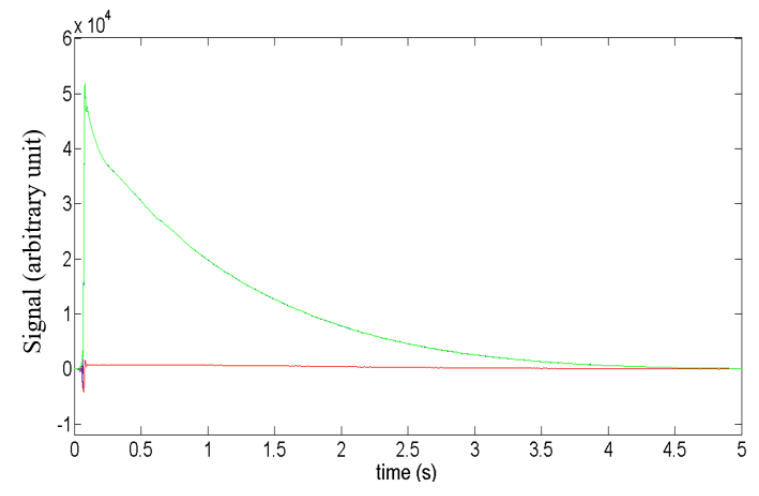

(a) Two-spin

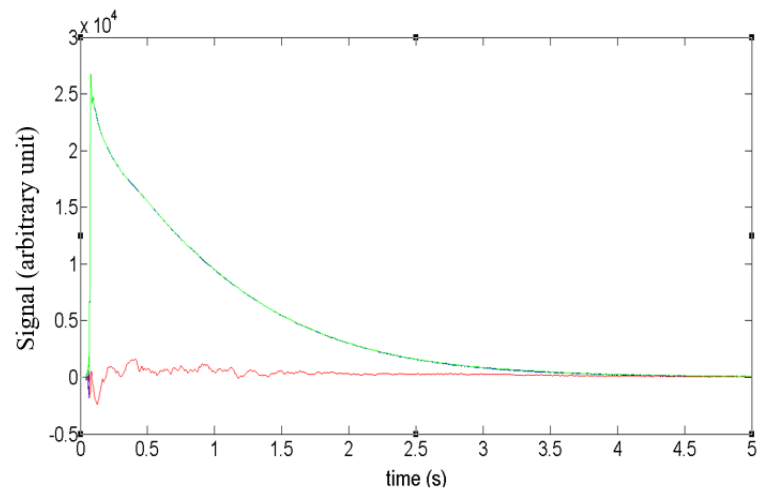

(b) One-spin

Figure 4.5: Experimental FID after demodulation with perfect synthesizer signal. No offset field is present. (a) and (b) are the demodulated FID signals of Figure.4.3(a) and Figure.4.3(b), respectively. The numerically generated perfect synthesizer signal $\cos \left(\omega_{0} t\right)$ is used for demodulation. In (a) and (b), the real, imaginary and absolute signals of the demodulated FIDs are in blue, red and green, respectively. The real and absolute signals overlap a lot due to the small imaginary signals. Both the FIDs decay due to the $T_{2}^{*}$. 


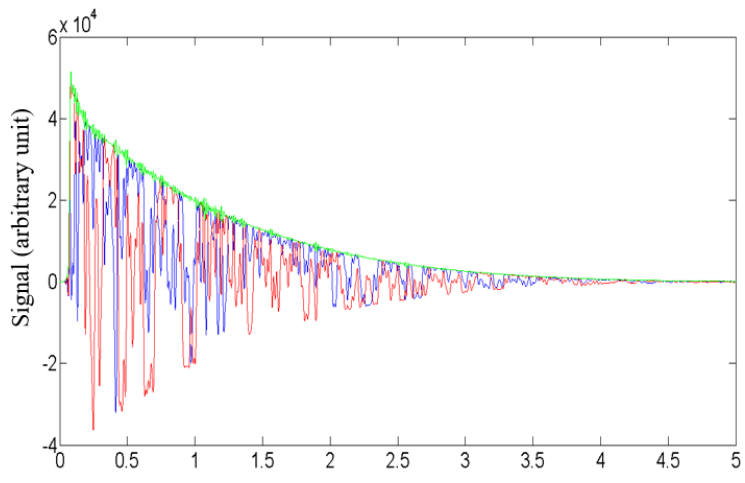

(a) Two-spin

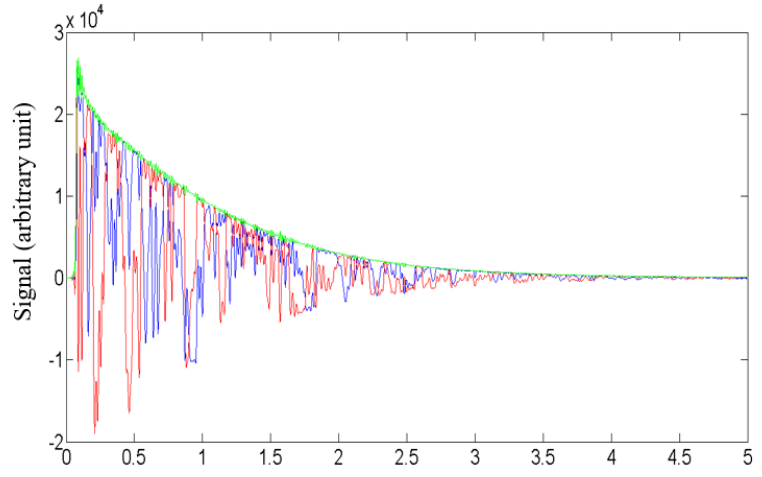

(b) One-spin

Figure 4.6: Experimental FID after demodulation with noisy synthesizer signal. No offset field is present. (a) and (b) are the demodulated FID signals of Figure.4.3(a) and Figure.4.3(b), respectively. The numerically generated perfect synthesizer signal $\cos \left(\omega_{0} t\right)$, with injected phase noise (Figure. 4.2), is used for demodulation. In (a) and (b), the real, imaginary and absolute signals of the demodulated FIDs are in blue, red and green, respectively. The real and imaginary signals of both the two spectra are very noisy because of the phase noise of the synthesizer signal. Both the FIDs decay due to the $T_{2}^{*}$.

is the usual case in real situations. Therefore, if permitted, averaging signals can reduce the effect of the noise. Figure.4.8 illustrates the average FIDs over 500 processings after demodulation with phase noise. There is signal loss due to averaging, but the noise is averaged to almost zero. The Fourier transform of the average FIDs shows clearly a single peak in both the two experiments, as shown in Figure.4.9. This means averaging is a good way for canceling noise if many copies of signal can be collected.

\subsubsection{Experiments with $B_{\alpha}$}

Next, we simulate the effect of $B_{\alpha}$ to the two-spin and one-spin systems by setting an offset frequency $\omega_{\alpha}$ to ${ }^{1} \mathrm{H}$ 's rotating frame when collecting FID data.

The FIDs in the $\mathrm{H}$ rotating frame of the two-spin and one-spin experiments are shown in Figure.4.10. In the two-spin experiment, apart from the real and imaginary parts, the amplitude of the signal oscillates, while in the one-spin experiment only real and imaginary parts of the signal oscillate. The Fourier transform spectra in Figure.4.11 shows the two 


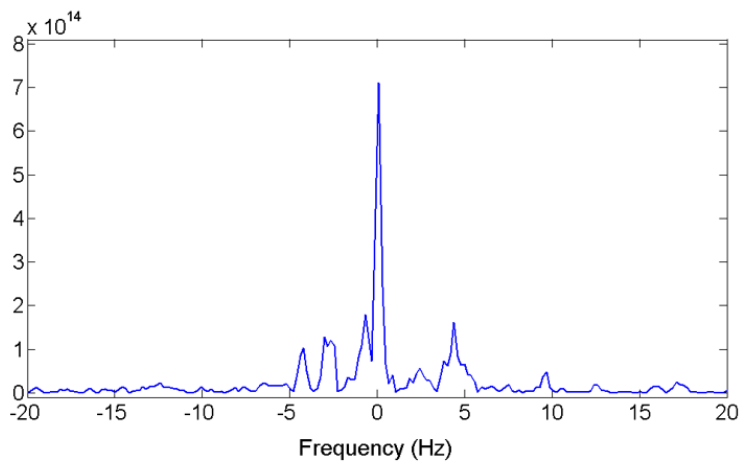

(a) Two-spin

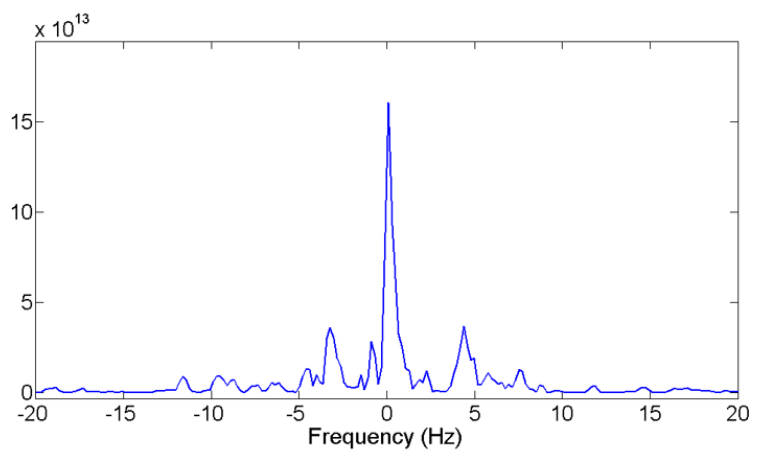

(b) One-spin

Figure 4.7: The Fourier transform spectra of the FIDs after demodulation with phase noise as in Figure. 4.6. The major peaks are at zero frequency because that there is no offset field.

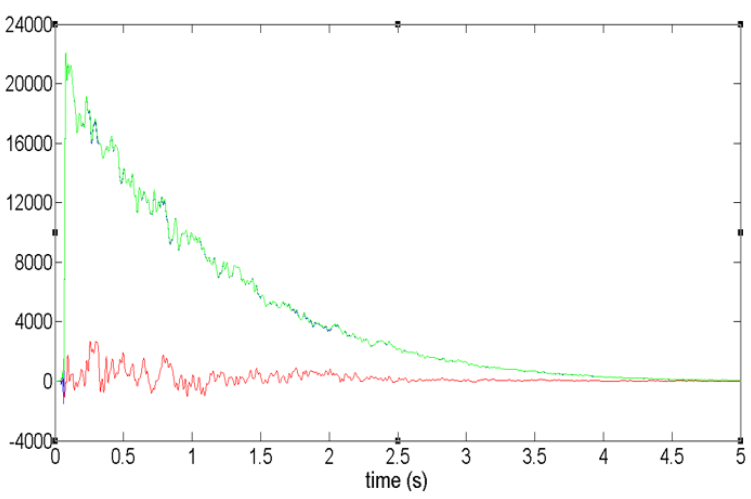

(a) Two-spin

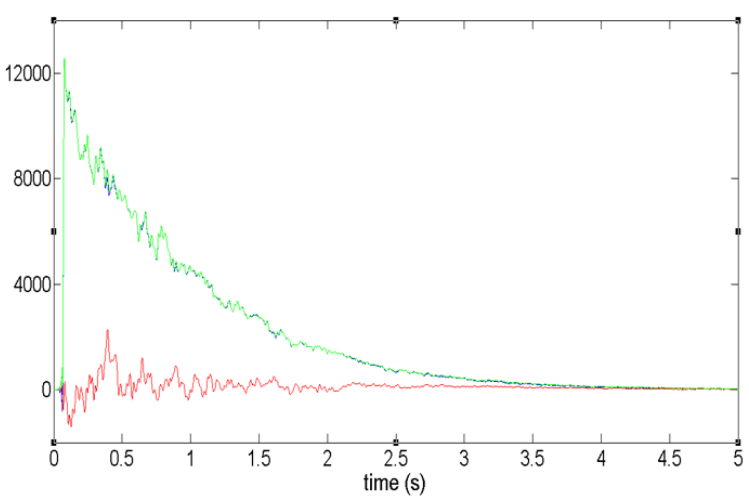

(b) One-spin

Figure 4.8: Average FIDs over 500 processings after demodulation with phase noise. (There is signal loss due to averaging, but the noise is averaged to almost zero.) 


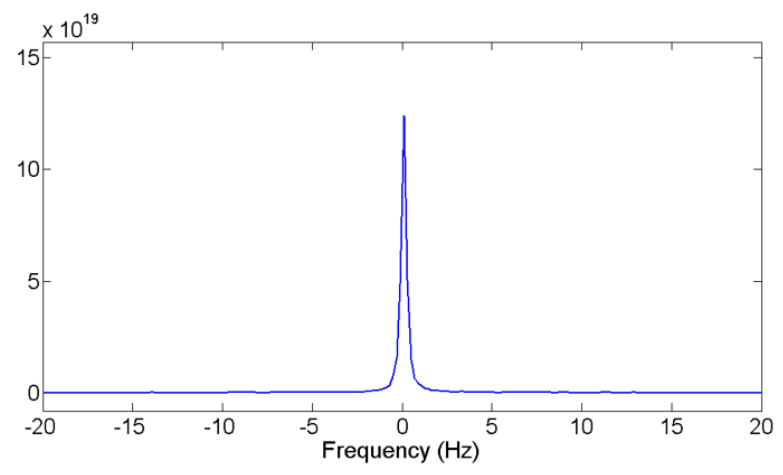

(a) Two-spin

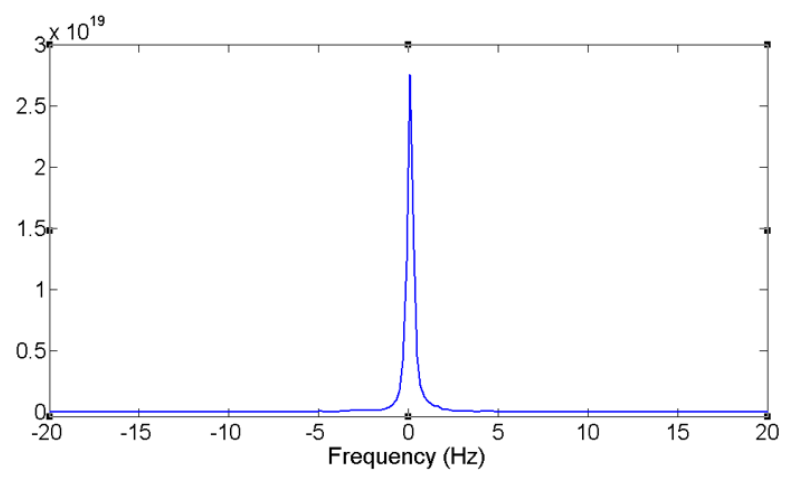

(b) One-spin

Figure 4.9: The Fourier transform spectra of the average FIDs as shown in Figure. 4.8.

frequencies in the two-spin experiment and one frequency in the one-spin experiment.

Figures 4.12(a) and (b) illustrate the FID signals after demodulation with the perfect synthesizer signal. It is clear that $\omega_{\alpha}$ manifests in the two-spin system as a modulation on the amplitude as derived from Eqs. (3.10) and (3.11), while as a phase modulation in the one-spin system.

When the synthesizer signal with phase noise is used for demodulation, as shown in Figures. 4.12(c) and (d), the phase noise does not affect the $\omega_{\alpha}$ amplitude modulation but interferes with the $\omega_{\alpha}$ phase modulation seriously. Thus the advantage of the two-spin system in detecting $B_{\alpha}$ is very clear. In presence of the phase noise, it is very difficult to extract $\omega_{\alpha}$ from the phase modulation of the one-spin system, but $\omega_{\alpha}$ can be obtained from the amplitude modulation of the two-spin system easily.

However, as shown in Figures. 4.14 and 4.15, averaging and Fourier transform are helpful in the case of zero-mean random noise for finding a constant offset frequency $\left(B_{\alpha}\right.$ field). But in cases where data is not enough for a high resolution Fourier transform, the signal in time domain becomes very important. And if averaging cannot be implemented, the amplitude information in the two-spin scheme is very useful.

In summary, in the presence of the synthesizer noise, it is very difficult to separate the effect of the offset magnetic field and the synthesizer noise, both of which are encoded in the phase information of the detected NMR signal after a Ramsey experiment. By using two single-qubit systems in two opposite magnetic fields, controlling and detecting using a same coil, the information of the offset magnetic field is encoded in the amplitude of the 


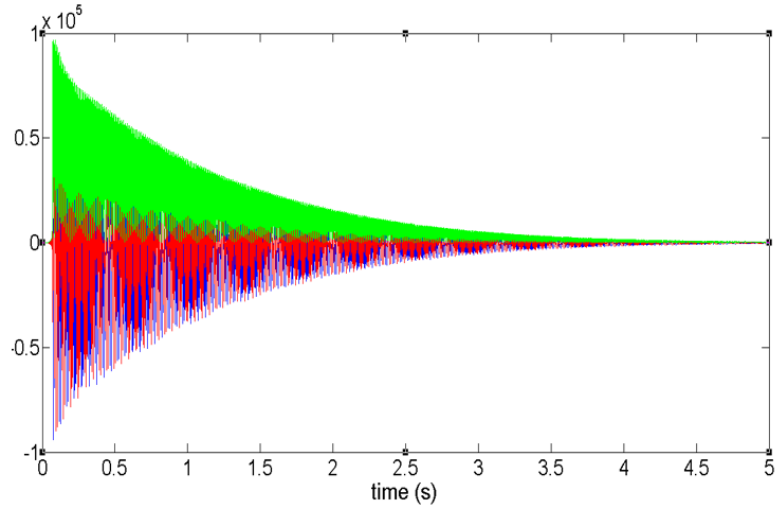

(a) Two-spin

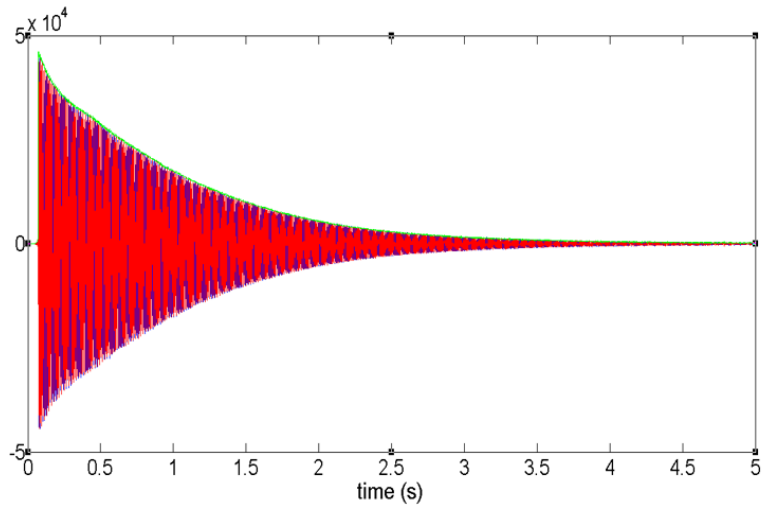

(b) One-spin

Figure 4.10: The FIDs in the $\mathrm{H}$ rotating frame of the two-spin and one-spin experiments when there is an offset field. Absolute, real and imaginary values are in green, blue and red, respectively. In the two-spin experiment, the amplitude of the signal oscillates, while in the one-spin experiment only real and imaginary parts of the signal oscillate. Those oscillations are around the frequency $\omega_{0}$. 


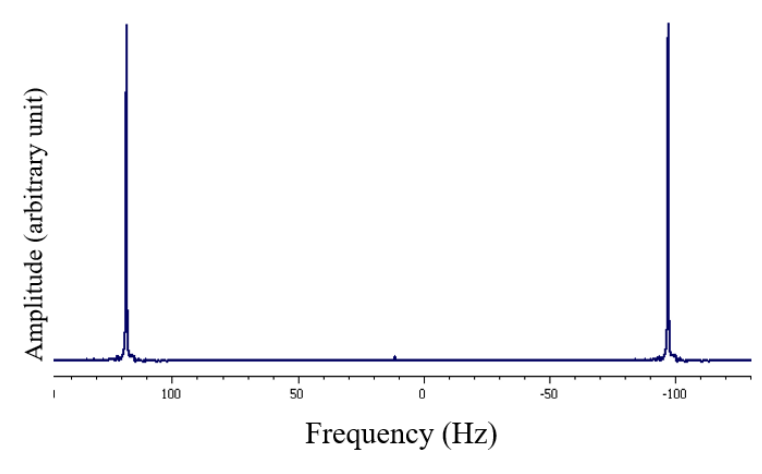

(a) Two-spin

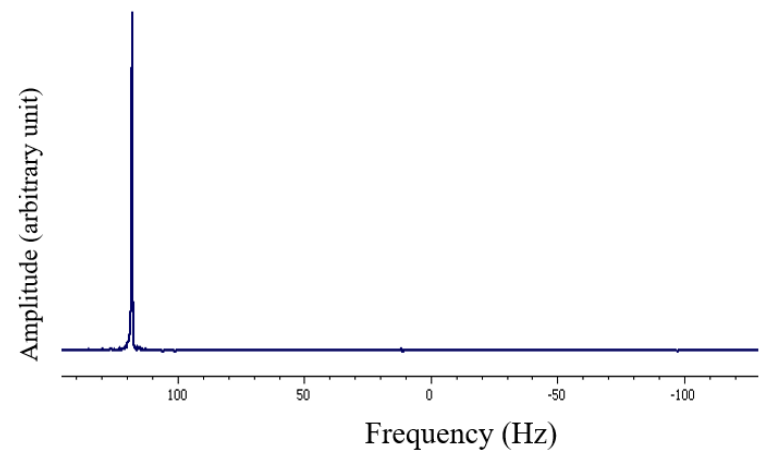

(b) One-spin

Figure 4.11: The Fourier transform spectra of the FIDs in Figure. 4.11. (a) is the spectrum of the experiment where two spins in two magnetic fields with $\pm \omega_{0}= \pm 107.6 \mathrm{~Hz}$ are simulated but the frequency shift to positive side compare to Figure. 4.4 (a). (b) is the spectrum of the experiment where one spin in one magnetic field with $\omega_{0}=107.6 \mathrm{~Hz}$ but also shift to the positive side compare to Figure. 4.4 (b)is simulated for comparison with (a). 

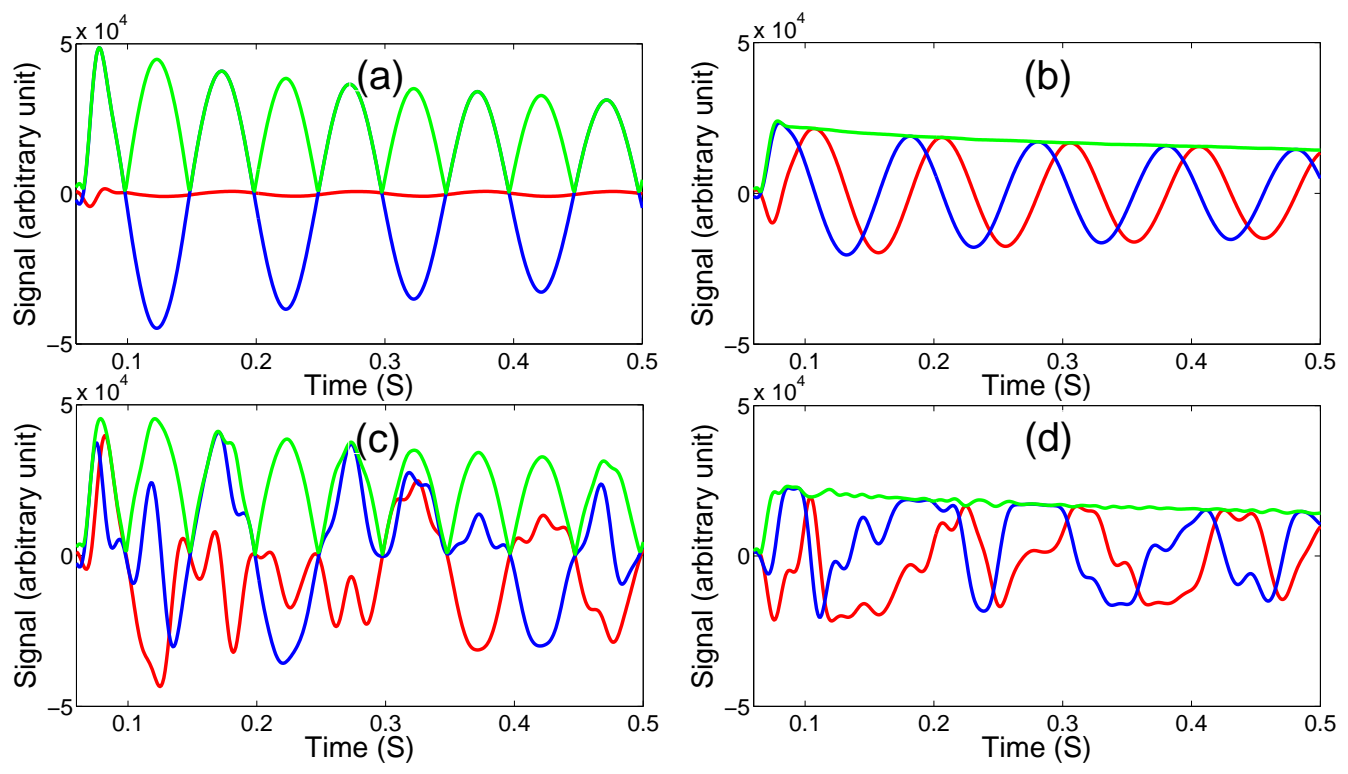

Figure 4.12: Experimental FID after demodulation in presence of an offset field $B_{\alpha}$ with $\omega_{\alpha}=10 \mathrm{~Hz}$. (a) and (c) are the FIDs from the experiment where two spins in two magnetic fields with $\pm \omega_{0}= \pm 107.6 \mathrm{~Hz}$ are simulated. (b) and (d) are the FIDs from the experiment where one spin in one magnetic field with $\omega_{0}=107.6 \mathrm{~Hz}$ is simulated for comparison. (a) and (b) are demodulated using the perfect synthesizer signal $\cos \left(\omega_{0} t\right)$. (c) and (d) are demodulated using the synthesizer signal with injected phase noise, $\cos \left(\omega_{0} t+\phi(t)\right)$. The real, imaginary and absolute signals of the demodulated FIDs are in blue, red and green, respectively. In (a), the absolute signal oscillates as $\left|\cos \left(\theta_{\alpha}\right)\right|$. In (c), although there is phase noise in demodulation, this oscillation remains. In (b), the real and imaginary signals oscillate as $\cos \left(\theta_{\alpha}\right)$ and $\sin \left(\theta_{\alpha}\right)$. After introduction of the phase noise, the oscillations are concealed by the noise as shown in $(d)$. 


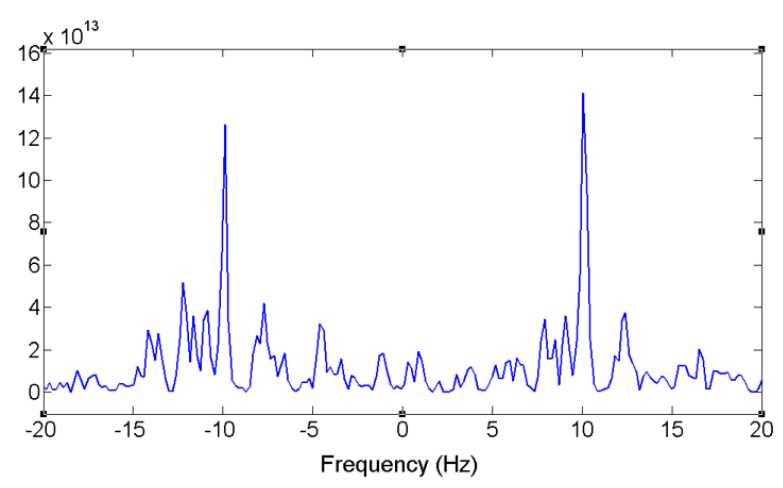

(a) Two-spin

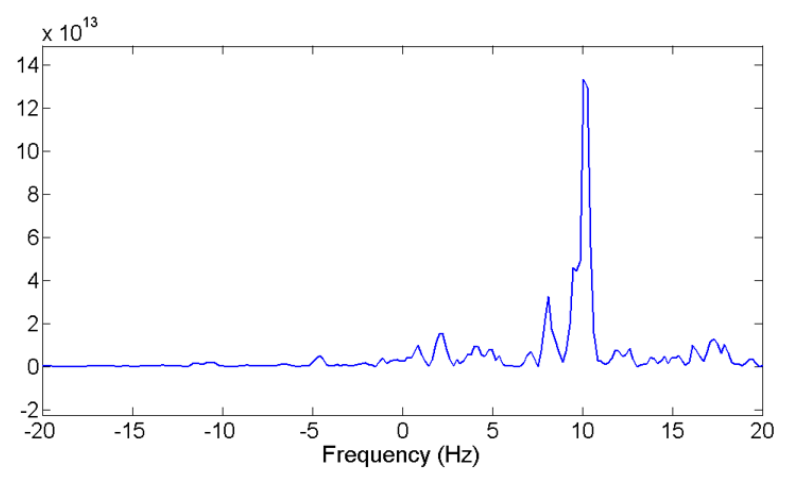

(b) One-spin

Figure 4.13: The Fourier transform spectra of the FIDs in Figure.4.12(c,d). The major peaks at $\pm 10 \mathrm{~Hz}$ come from the offset field, and the noisy small peaks come from the synthesizer phase noise.

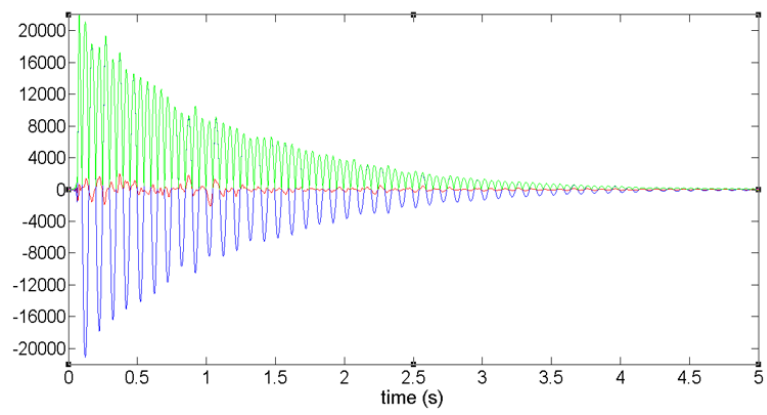

(a) Two-spin

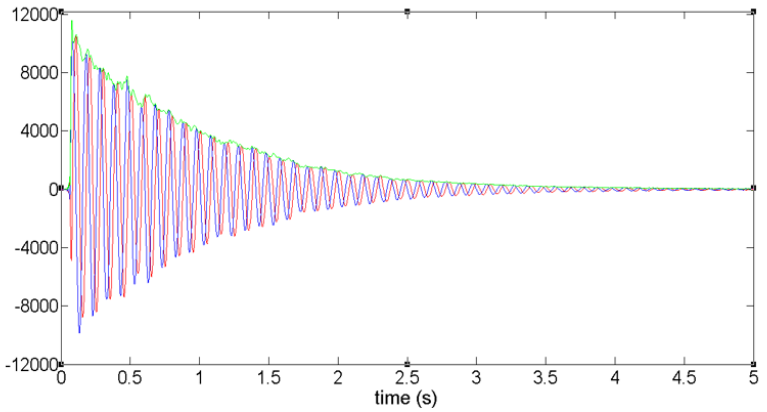

(b) One-spin

Figure 4.14: The average of 500 copies of the FIDs after demodulation with phase noise. (There is signal loss due to averaging, but the noise is averaged to almost zero.) 


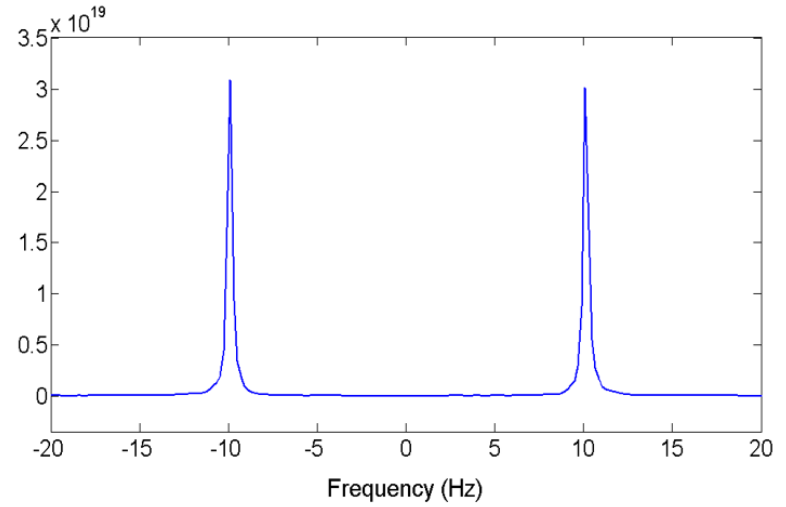

(a) Two-spin

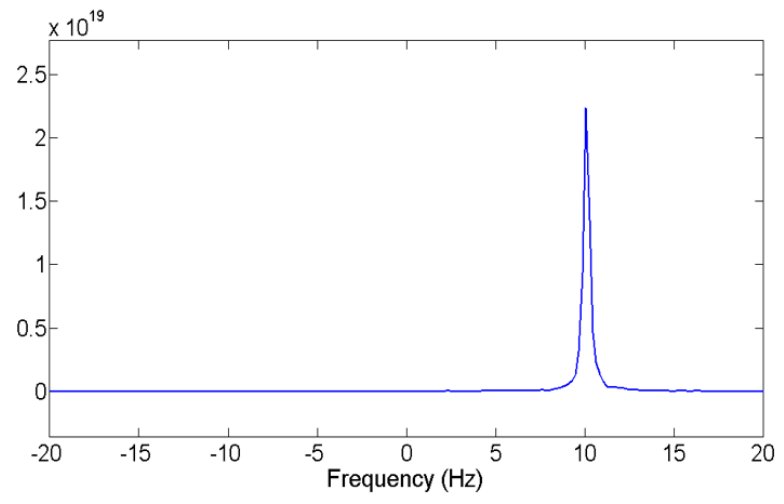

(b) One-spin

Figure 4.15: The Fourier transform spectra of the average FIDs as shown in Figure. 4.14. The noise disappears and only the major peaks remain. The major peaks have a frequency of $\pm \omega_{0}$.

detected NMR signal. Therefore, it is separated from the effect of the synthesizer noise, and thus the measurement accuracy is improved. 


\section{Chapter 5}

\section{Conclusions and Discussions}

Ramsey fringe experiments can be used to measure an offset magnetic field in the environment. However, it is very difficult to separate the effect of the environmental magnetic field and the synthesizer phase noise, of which both are encoded in the phase information of the detected spin signal if a one-spin system is used. We proposed to use two singlespin systems in opposite magnetic fields to detect the environmental field. The two spins are controlled using the same synthesizer signal. They can be controlled by two antennas (two-antenna configuration) or by the same antenna (one-antenna configuration). The synthesizer phase noise affects the two spins oppositely. In the two-antenna configuration, the phase noise of the two spins can cancel each other and the net effect of the environmental field can be detected. In the one-antenna configuration, the effects of the environmental field and the phase noise are encoded in the amplitude and phase of the detected signal separately. Therefore, the environmental field can also be detected accurately.

There are potential challenges in both the two configurations. As we discussed above, the two-antenna configuration needs additional components to split the synthesizer signal to the two antennas. The transmission line should be designed to be symmetric carefully so that there is no additional difference between the two spins to avoid reducing accuracy. Although the one-antenna configuration has no risk of inducing additional errors, the technical challenge is more severe, i.e., how to realize two opposite magnetic fields within the range of a single antenna. The field cannot be too weak, otherwise, the rotation wave approximation will fail and thus this method may fail. Besides, the one-antenna configuration utilizes the quadrature detection technique and thus has a less general application than the two-antenna configuration.

In spite of the technical challenges, the proposed scheme is still very attractive for 
improving accuracy in quantum sensing as the synthesizer phase noise has become an important issue because of high qubit qualities and demands for miniaturization of quantum devices. Although this work utilizes an NMR system for demonstration, the basic idea of the scheme, using two spins in opposite static magnetic fields, can also be applied to other quantum platforms where the Zeeman interaction dominates the Hamiltonian, and a synthesizer and thus the synthesizer phase noise is present. 


\section{References}

[1] R. Feynman. Int J Theor Phys, 21: 467, 1982.

[2] P. Benioff. Quantum Mechanical Models of Turing Machines That Dissipate No Energy. Physical review letters, 48: 1581, 1982.

[3] D.Deutsch, and R.Penrose. Quantum theory, the Church Turing principle and the universal quantum computer. Proceedings of the Royal Society of London. A. Mathematical and Physical Sciences, 1985.

[4] P. Shor. Polynomial-Time Algorithms for Prime Factorization and Discrete Logarithms on a Quantum Computer. SIAM J. Comput., 26(5): 1484-1509, 1999.

[5] Grover L K. Quantum mechanics helps in searching for a needle in a haystack. Physical review letters, 79(2): 325, 1997.

[6] Grover L K. Quantum computers can search arbitrarily large databases by a single query. Physical review letters, 79(23): 4709, 1997.

[7] Grover L K. A framework for fast quantum mechanical algorithms. Proceedings of Proceedings of the thirtieth annual ACM symposium on Theory of computing. ACM, 53-62, 1998.

[8] Boyer M, Brassard G, and Hoyer P, et al. Tight bounds on quantum searching. Fortschritte der Physik, 46(4-5):493-506, 1998.

[9] Long G L, Li Y S, and Zhang W L, et al. Phase matching in quantum searching. Physics Letters A, 262(1):27-34, 1999.

[10] Zalka C. Grover's quantum searching algorithm is optimal. Physical review A, 60(4): 2746, 1999. 
[11] Long G L. Grover algorithm with zero theoretical failure rate. Physical review A, 64(2):022307, 2001.

[12] Bruschweiler R. Novel strategy for database searching in spin Liouville space by NMR ensemble computing. Physical review letters, 85(22):4815, 2000.

[13] Brassard G, Hoyer P, and Tapp A. Quantum counting. Proceedings of Automata, Languages and Programming. Berlin: Springer, 820-831, 1998.

[14] Abrams D S, and Lloyd S. Quantum algorithm providing exponential speed increase for finding eigenvalues and eigenvectors. Physical review letters, 83(24):5162, 1999.

[15] Spector L, Barnum H, and Bernstein H J, et al. Finding a better-than-classical quantum AND/OR algorithm using genetic programming. Proceedings of Proceedings of the Congress on Evolutionary Computation, 3: 2239-2246, 1999.

[16] Chuang I L. Quantum algorithm for distributed clock synchronization. Physical review letters, 85(9):2006, 2000.

[17] Han K H, and Kim J H. Genetic quantum algorithm and its application to combinatorial optimization problem. Proceedings of Evolutionary Computation, 2: 1354-1360, 2000 .

[18] Farhi E, Goldstone J, and Gutmann S, et al. A quantum adiabatic evolution algorithm applied to random instances of an NP-complete problem. Science, 292(5516):472-475, 2001.

[19] Shenvi N, Kempe J, and Whaley K B. Quantum random-walk search algorithm. Physical review A, 67(5):052307, 2003.

[20] Kuperberg G. A subexponential-time quantum algorithm for the dihedral hidden subgroup problem. SIAM Journal on Computing, 35(1):170-188, 2005.

[21] Ambainis A. Quantum walk algorithm for element distinctness. SIAM Journal on Computing, 37(1):210-239, 2007.

[22] Magniez F, Santha M, and Szegedy M. Quantum algorithms for the triangle problem. SIAM Journal on Computing, 37(2):413-424, 2007.

[23] Aharonov D, Jones V, and Landau Z. A polynomial quantum algorithm for approximating the Jones polynomial. Algorithmica, 55(3):395-421, 2009. 
[24] Harrow A W, Hassidim A, and Lloyd S. Quantum algorithm for linear systems of equations. Physical review letters, 103(15):150502, 2009.

[25] Reichardt B, and Spalek R. Span-Program-Based Quantum Algorithm for Evaluating Formulas. Theory OF Computing, 8(1):291-319, 2012.

[26] Jordan S P, Lee K S M, and Preskill J. Quantum algorithms for quantum field theories. Science, 336(6085):1130-1133, 2012.

[27] Negrevergne C, Somma R, and Ortiz G, et al. Liquid-state NMR simulations of quantum many body problems. Physical review A, 71(3):032344, 2005.

[28] Somaroo S, Tseng C H, and Havel T F, et al. Quantum Simulations on a Quantum Computer. Physical review letters, 82(26):5381, 1999.

[29] Greiner M, Mandel O, and Esslinger T, et al. Quantum phase transition from a superfluid to a Mott insulator in a gas of ultracold atoms. Nature, 415(6867):39-44, 2002 .

[30] Peng X, Du J, and Suter D. Quantum phase transition of ground-state entanglement in a Heisenberg spin chain simulated in an NMR quantum computer. Physical review A, 71(1):012307, 2005.

[31] Zhang J, Peng X, and Rajendran N, et al. Detection of quantum critical points by a probe qubit. Physical review letters, 100(10):100501, 2008.

[32] Friedenauer A, Schmitz H, and Glueckert J T, et al. Simulating a quantum magnet with trapped ions. Nature Physics, 4(10):757-761, 2008.

[33] Gerritsma R, Kirchmair G, and Zahringer F, et al. Quantum simulation of the Dirac equation. Nature, 463(7277):68-71, 2010.

[34] Deutsch D. Quantum computational networks. Proceedings of the Royal Society of London. A. Mathematical and Physical Sciences, 425(1868):73-90, 1989.

[35] Kitaev A Y. Fault-tolerant quantum computation by anyons. Annals of Physics, 303(1):2-30, 2003.

[36] Kitaev A. Anyons in an exactly solved model and beyond. Annals of Physics, 321(1):2$111,2006$. 
[37] Freedman M H, Larsen M, and Wang Z. A Modular Functor Which is Universal for Quantum Computation. Communications in Mathematical Physics, 227(3):605-622, 2002 .

[38] Freedman M H, Kitaev A, and Wang Z. Simulation of Topological Field Theories by Quantum Computers. Communications in Mathematical Physics, 227(3):587-603, 2002 .

[39] Nayak C, Simon S H, and Stern A, et al. Non-Abelian anyons and topological quantum computation. Reviews of Modern Physics, 80(3):1083, 2008.

[40] Mizel A, Lidar D A, and Mitchell M. Simple proof of equivalence between adiabatic quantum computation and the circuit model. Physical review letters, 99(7):070502, 2007.

[41] Zagoskin A. Quantum engineering: theory and design of quantum coherent structures. Cambridge:Cambridge University press, 2011.

[42] Raussendorf R, and Briegel H J. A One-Way Quantum Computer. Physical Review Letters, 86:5188-5191, 2001.

[43] Shor P W. Scheme for reducing decoherence in quantum computer memory. Physical review A, 52(4):R2493, 1995.

[44] Steane A. Multiple-particle interference and quantum error correction. Proceedings of the Royal Society of London. Series A: Mathematical, Physical and Engineering Sciences, 452(1954):2551-2577, 1996.

[45] Knill E, Laflamme R, and Zurek W H. Resilient quantum computation. Science, 279(5349):342-345, 1998.

[46] H. Ball, W. D. Oliver, and M. J Biercuk, The role of master clock stability in quantum information processing, npj Quantum Information 2, 16033, 2016.

[47] D. Gottesman. An Introduction to Quantum Error Correction and Fault-Tolerant Quantum Computation, Quantum Information Science and Its Contributions to Mathematics, Proceedings of Symposia in Applied Mathematics, 68:13-58, 2010.

[48] Knill E, Laflamme R, and Zurek W H. Resilient quantum computation: error models and thresholds. Proceedings of the Royal Society of London. Series A: Mathematical, Physical and Engineering Sciences, 454(1969):365-384, 1998. 
[49] Knill E. Quantum computing with realistically noisy devices. Nature, 434(7029):39-44, 2005.

[50] Aliferis P, Gottesman D, Preskill J. Quantum accuracy threshold for concatenated distance-3 codes. Quant. Inf. Comput., 6:97-165, 2006.

[51] Aliferis P, Gottesman D, Preskill J. Accuracy threshold for postselected quantum computation. Quant. Inf. Comput., 8:181-244, 2008.

[52] Gottesman D. Stabilizer codes and quantum error correction[D]. Pasadena: California Inst. of Technol., 1997.

[53] C.L. Degen, F. Reinhard, and P. Cappellaro. Quantum sensing. Rev. Mod. Phys., 89, $035002,2017$.

[54] R. C. Jaklevic, J. Lambe, J. E. Mercereau, and A. H. Silver. Macroscopic Quantum Interference in Superconductors. Phys. Rev. 140, A1628, 1965.

[55] I. K. Kominis, T. W. Kornack, J. C. Allred, and M. V. Romalis. A subfemtotesla multichannel atomic magnetometer.Nature, 422:596-599, 2003.

[56] P. O. Schmidt, T. Rosenband, C. Langer, W. M. Itano, J. C. Bergquist, D. J. Wineland.Spectroscopy Using Quantum Logic.Science,309:749-752,2005.

[57] Ashok Ajoy and Paola Cappellaro. Mixed-state quantum transport in correlated spin networks. Physical review A 85, 042305, 2012.

[58] W C Campbell, and P Hamilton. Rotation sensing with trapped ions. Journal of Physics B: Atomic, Molecular and Optical Physics, 50, 2017.

[59] R. Barends, J. Kelly, A. Megrant, A. Veitia, D. Sank, E. Jeffrey, T. C. White, J. Mutus, A. G. Fowler, B. Campbell, Y. Chen, Z. Chen, B. Chiaro, A. Dunsworth, C. Neill, P. O'Malley, P. Roushan, A. Vainsencher, J. Wenner, A. N. Korotkov, A. N. Cleland, and J. M. Martinis, Superconducting quantum circuits at the surface code threshold for fault tolerance, Nature 508 (7497):500-503, 2014.

[60] M. Veldhorst, J. C. C. Hwang, C. H. Yang, A. W. Leenstra, B. de Ronde, J. P. Dehollain, J. T. Muhonen, F. E. Hudson, K. M. Itoh, A. Morello, and A. S. Dzurak, An addressable quantum dot qubit with fault-tolerant control-fidelity, Nature Nano. 9:981-985, 2014. 
[61] X. Rong, J Geng, F. Shi, Y. Liu, K. Xu, W. Ma, F. Kong, Z. Jiang, Y. Wu, J. $\mathrm{Du}$, Experimental fault-tolerant universal quantum gates with solid-state spins under ambient conditions, Nature Communications 6, 8748, 2015.

[62] A. Bermudez, X. Xu, R. Nigmatullin, J. O'Gorman, V. Negnevitsky, P. Schindler, T. Monz, U. G. Poschinger, C. Hempel, J. Home, F. Schmidt-Kaler, M. Biercuk, R. Blatt, S. Benjamin, and M. Müller, Assessing the Progress of Trapped-Ion Processors Towards Fault-Tolerant Quantum Computation, Phys. Rev. X 7, 041061, 2017.

[63] J. Sastrawan, C. Jones, I. Akhalwaya, H. Uys, and M. J. Biercuk, Analytically exploiting noise correlations inside the feedback loop to improve locked-oscillator performance, Physical Review E 94, 022204, 2016.

[64] G. Gordon, G. Kurizki, and D. A. Lidar, Optimal dynamical decoherence control of a qubit, Phys. Rev. Lett. 101, 010403, 2008.

[65] K. Khodjasteh and L. Viola, Dynamically error-corrected gates for universal quantum computation, Phys. Rev. Lett. 102, 080501, 2009.

[66] K. Khodjasteh, D. A. Lidar, and L. Viola, Arbitrarily accurate dynamical control in open quantum systems, Phys. Rev. Lett. 104, 090501, 2010.

[67] A. M. Souza, G. A. Álvarez, and D. Suter, Robust dynamical decoupling for quantum computing and quantum memory, Phys. Rev. Lett. 106, 240501, 2011.

[68] N. Khaneja, T. Reiss, C. Kehlet, T. Schulte-Herbrüggen, and S. J. Glaser, Journal of Magnetic Resonance 172, 296-305, 2005.

[69] D. Layden and P. Cappellaro, Error-corrected quantum sensing with parallel signal and noise, arXiv.1708.06729, 2017.

[70] Purcell E M, Torrey H C, Pound R V. Resonance absorption by nuclear magnetic moments in a solid. Physical review, 69(1-2):37, 1946.

[71] Bloch F. Nuclear induction. Physical review, 70(7-8):460, 1946.

[72] Vandersypen L M K, Chuang I L. NMR techniques for quantum control and computation.Reviews of Modern Physics, 76:1037-1069, 2005.

[73] D. G. Cory, A. F. Fahmy, and T. F. Havel. Ensemble quantum computing by NMRspectroscopy. Proc. Nat. Ac. of Sci. USA, 94:1634-1639, 1997. 
[74] N. A. Gershenfeld and I. L. Chuang. Bulk spin resonance quantum computation. Science, 275:350- 356, 1997.

[75] Vandersypen L M K, Chuang I L. NMR techniques for quantum control and computation.Reviews of Modern Physics, 76:1037-1069, 2005.

[76] N.F. Ramsey. A molecular beam resonance method with separated oscillating fields. Phys. Rev., 78:695, 1950.

[77] S. Sheldon, "Optimal control in an open quantum system : selecting DNP pathways in an electron-nuclear system", Ph.D dissertation, Department of Nuclear Science and Engineering, Massachusetts Institute of Technology, Massachusetts. 